\title{
Two-loop fermionic corrections to massive Bhabha scattering
}

\author{
Stefano Actis ${ }^{\mathrm{a}, *}$, Michał Czakon ${ }^{\mathrm{b}, \mathrm{c}}$, Janusz Gluza ${ }^{\mathrm{d}}$, Tord Riemann ${ }^{\mathrm{a}}$ \\ a Deutsches Elektronen-Synchrotron, DESY, Platanenallee 6, D-15738 Zeuthen, Germany \\ ${ }^{\mathrm{b}}$ Institut für Theoretische Physik und Astrophysik, Universität Würzburg, Am Hubland, D-97074 Würzburg, Germany \\ c Institute of Nuclear Physics, NCSR "DEMOKRITOS", 15310 Athens, Greece \\ d Institute of Physics, University of Silesia, Uniwersytecka 4, PL-40007 Katowice, Poland
}

Received 9 May 2007; accepted 27 June 2007

Available online 4 July 2007

\begin{abstract}
We evaluate the two-loop corrections to Bhabha scattering from fermion loops in the context of pure quantum electrodynamics. The differential cross section is expressed by a small number of master integrals with exact dependence on the fermion masses $m_{e}, m_{f}$ and the Mandelstam invariants $s, t, u$. We determine the limit of fixed scattering angle and high energy, assuming the hierarchy of scales $m_{e}^{2} \ll m_{f}^{2} \ll s, t, u$. The numerical result is combined with the available non-fermionic contributions. As a by-product, we provide an independent check of the known electron-loop contributions.

(c) 2007 Elsevier B.V. All rights reserved.
\end{abstract}

\section{Introduction}

Bhabha scattering is one of the processes at $e^{+} e^{-}$colliders with the highest experimental precision and represents an important monitoring process. A notable example is its expected role for the luminosity determination at the future International Linear Collider ILC by measuring smallangle Bhabha-scattering events at center-of-mass energies ranging from about $100 \mathrm{GeV}$ (Giga-Z collider option) to several TeV. Moreover, the large-angle region is relevant at colliders operat-

\footnotetext{
* Corresponding author.

E-mail address: stefano.actis@desy.de (S. Actis).
} 
ing at $1-10 \mathrm{GeV}$. For some applications a full two-loop calculation of the QED contributions is mandatory. ${ }^{1}$

A large class of QED two-loop corrections was determined in the seminal work of [2]. Later, the complete two-loop corrections in the limit of zero electron mass were obtained in [3] thanks to the fundamental results of $[4,5]$. However, this result cannot be immediately applied, since the available Monte Carlo programs (see, e.g., [6-13]) employ a small, but non-vanishing electron mass. The $\alpha^{2} \ln \left(s / m_{e}^{2}\right)$ terms due to double boxes were derived from [3] by the authors of [14], and the close-to-complete two-loop result in the ultra-relativistic limit was finally obtained in $[15,16]$. Note that the diagrams with fermion loops have not been covered by this approach. The virtual and real components involving electron loops could be added exactly in $[17,18]$. The non-approximated analytical expressions for all two-loop corrections, except for double-box diagrams and for those with loops from heavier-fermion generations, can be found in [19]. For a comprehensive investigation of the full set of the massive two-loop QED corrections, including double-box diagrams, we refer to [20-24]. The evaluation of the contributions from massive non-planar double box diagrams remains open so far.

In order to add another piece to the complete two-loop prediction for the Bhabha-scattering cross section in QED, we evaluate here the so-far lacking diagrams containing heavy-fermion loops. The cross-section correction is expressed by a small number of scalar master integrals, where the exact dependence on the masses of the fermions and the Mandelstam variables $s, t$ and $u$ is retained. In a next step, we assume a hierarchy of scales, $m_{e}^{2} \ll m_{f}^{2} \ll s, t$, where $m_{e}$ is the electron mass and $m_{f}$ is the mass of a heavier fermion. We derive explicit results neglecting terms suppressed by positive powers of $m_{e}^{2} / m_{f}^{2}, m_{e}^{2} / x$ and $m_{f}^{2} / x$, where $x=s, t, u$. This highenergy approximation describes the influence of muons and $\tau$ leptons and proves well-suited for practical applications. In addition, we provide an independent cross-check of the exact analytical results of [17] (we used the files provided at [25] for comparison) for $m_{f}=m_{e}$.

The article is organized as follows. In Section 2 we introduce our notations and outline the calculation and in Section 3 we discuss the solution for each class of diagrams. In Section 4 we reproduce the complete result for the corrections from heavier fermions in analytic form and perform the numerical analysis. Section 5 contains the summary, and additional material on the master integrals is collected in Appendix A.

\section{Expansion of the cross section}

We consider the Bhabha-scattering process,

$$
e^{-}\left(p_{1}\right)+e^{+}\left(p_{2}\right) \rightarrow e^{-}\left(p_{3}\right)+e^{+}\left(p_{4}\right),
$$

and introduce the Mandelstam invariants $s, t$ and $u$,

$$
\begin{aligned}
& s=\left(p_{1}+p_{2}\right)^{2}=4 E^{2}, \\
& t=\left(p_{1}-p_{3}\right)^{2}=-4\left(E^{2}-m_{e}^{2}\right) \sin ^{2} \frac{\theta}{2}, \\
& u=\left(p_{1}-p_{4}\right)^{2}=-4\left(E^{2}-m_{e}^{2}\right) \cos ^{2} \frac{\theta}{2},
\end{aligned}
$$

where $m_{e}$ is the electron mass, $E$ is the incoming-particle energy in the center-of-mass frame and $\theta$ is the scattering angle. In addition, $s+t+u=4 m_{e}^{2}$.

\footnotetext{
1 Note that leading two-loop effects in the electroweak Standard Model were already incorporated in [1].
} 


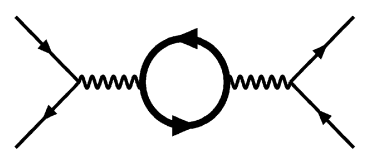

$1 \mathrm{a}$

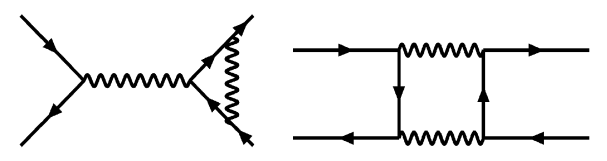

$1 \mathrm{~b}$

$1 \mathrm{c}$

Fig. 1. Classes of Bhabha-scattering one-loop diagrams. A thin fermion line represents an electron, a thick one can be any fermion. The full set of graphs can be obtained through proper permutations. We refer to [26] for the reproduction of the full set of graphs.

In the kinematical region $m_{e}^{2} \ll s, t, u$ the leading-order (LO) differential cross section with respect to the solid angle $\Omega$ reads as

$$
\frac{d \sigma^{\mathrm{LO}}}{d \Omega}=\frac{\alpha^{2}}{s}\left[\frac{1}{s^{2}}\left(\frac{s^{2}}{2}+t^{2}+s t\right)+\frac{1}{t^{2}}\left(\frac{t^{2}}{2}+s^{2}+s t\right)+\frac{1}{s t}(s+t)^{2}\right],
$$

where $\alpha$ is the fine-structure constant. At higher orders in perturbation theory we write an expansion in $\alpha$,

$$
\frac{d \sigma}{d \Omega}=\frac{d \sigma^{\mathrm{LO}}}{d \Omega}+\left(\frac{\alpha}{\pi}\right) \frac{d \sigma^{\mathrm{NLO}}}{d \Omega}+\left(\frac{\alpha}{\pi}\right)^{2} \frac{d \sigma^{\mathrm{NNLO}}}{d \Omega}+\mathcal{O}\left(\alpha^{5}\right) .
$$

Here $d \sigma^{\mathrm{NLO}}$ and $d \sigma^{\mathrm{NNLO}}$ summarize the next-to-leading order (NLO) and next-to-next-toleading order (NNLO) corrections to the differential cross section. In the following it will be understood that we consider only components generated by diagrams containing one or two fermion loops.

\subsection{NLO differential cross section}

The NLO term follows from the interference of the one-loop vacuum-polarization diagrams of class 1a (see Fig. 1) with the tree-level amplitude,

$$
\begin{aligned}
\frac{d \sigma^{\mathrm{NLO}}}{d \Omega}= & \frac{d \sigma^{1 \mathrm{a} \times \text { tree }}}{d \Omega} \\
= & \frac{\alpha^{2}}{s}\left\{\frac{1}{s^{2}}\left(\frac{s^{2}}{2}+t^{2}+s t\right) 2 \sum_{f} Q_{f}^{2} \operatorname{Re}\left[\Pi_{f}^{(1)}(s)\right]\right. \\
& +\frac{1}{t^{2}}\left(\frac{t^{2}}{2}+s^{2}+s t\right) 2 \sum_{f} Q_{f}^{2} \operatorname{Re}\left[\Pi_{f}^{(1)}(t)\right] \\
& \left.+\frac{1}{s t}(s+t)^{2} \sum_{f} Q_{f}^{2} \operatorname{Re}\left[\Pi_{f}^{(1)}(s)+\Pi_{f}^{(1)}(t)\right]\right\} .
\end{aligned}
$$

Here $\Pi_{f}^{(1)}(x)$ is the renormalized one-loop vacuum-polarization function and the sum over $f$ runs over the massive fermions, e.g., the electron $(f=e)$, the muon $(f=\mu)$, the $\tau$ lepton ( $f=\tau) . Q_{f}$ is the electric-charge quantum number, $Q_{f}=-1$ for leptons.

In this paper we will focus on asymptotic expansions in the high-energy limit. In order to fix our normalizations explicitly, we reproduce here the exact result for $\Pi_{f}^{(1)}(x)$ in dimensional regularization. Adding $\Pi_{f}^{(1) c t}(x)$, the counterterm contribution in the on-mass-shell scheme (see 
the following discussion in Section 2.3), to $\Pi_{f}^{(1) u n}(x)$, the unrenormalized one-loop vacuum polarization function, we get

$$
\begin{aligned}
& \Pi_{f}^{(1)}(x)=\Pi_{f}^{(1) u n}(x)+\Pi_{f}^{(1) c t}(x), \\
& \Pi_{f}^{(1) u n}(x)=\frac{1}{2(D-1)}\left[2(D-2) \frac{1}{x} A_{0}\left(m_{f}\right)-\left(D-2+4 \frac{m_{f}^{2}}{x}\right) B_{0}\left(x, m_{f}\right)\right], \\
& \Pi_{f}^{(1) c t}(x)=\frac{1}{3} F_{\epsilon}\left(\frac{m_{e}^{2}}{m_{f}^{2}}\right)^{\epsilon}\left(\frac{1}{\epsilon}+\frac{\zeta_{2}}{2} \epsilon\right),
\end{aligned}
$$

where $\epsilon=(4-D) / 2$ and $D$ is the number of space-time dimensions. The normalization factor is

$$
F_{\epsilon}=\left(\frac{m_{e}^{2} \pi e^{\gamma_{E}}}{\mu^{2}}\right)^{-\epsilon},
$$

$\mu$ is the 't Hooft mass unit and $\gamma_{E}$ is the Euler-Mascheroni constant. Standard one-loop integrals appearing in Eq. (2.8) are defined by

$$
\begin{aligned}
& A_{0}(m)=\frac{\mu^{4-D}}{i \pi^{2}} \int d^{D} k \frac{1}{k^{2}-m^{2}}, \\
& B_{0}\left(p^{2}, m\right)=\frac{\mu^{4-D}}{i \pi^{2}} \int d^{D} k \frac{1}{\left(k^{2}-m^{2}\right)\left[(k+p)^{2}-m^{2}\right]} .
\end{aligned}
$$

Note that master integrals with 1 lines and an internal scale $m$ were derived in [22,26] setting $m=1$. For the present computation we introduce a scaling by a factor $m_{f}^{D-2 l}$ and we get

$$
\begin{aligned}
& A_{0}\left(m_{f}\right)=F_{\epsilon}\left(\frac{m_{e}^{2}}{m_{f}^{2}}\right)^{\epsilon} m_{f}^{2} \mathrm{~T} 111 \mathrm{~m}, \\
& B_{0}\left(x, m_{f}\right)=F_{\epsilon}\left(\frac{m_{e}^{2}}{m_{f}^{2}}\right)^{\epsilon} \operatorname{SE} 212 \mathrm{~m}[\mathrm{x}] .
\end{aligned}
$$

In the small-mass limit, $A_{0}$ vanishes (the result for T111m can be read in Eq. (4) of [22]), and the one-loop self-energy ${ }^{2}$ reads as

$$
\operatorname{SE} 212 \mathrm{~m}[\mathrm{x}]=\frac{1}{\epsilon}+2+L_{f}(x)+\epsilon\left[4-\frac{\zeta_{2}}{2}+2 L_{f}(x)+\frac{1}{2} L_{f}^{2}(x)\right],
$$

where we introduced the short-hand notation for logarithmic functions (in our conventions the logarithm has a cut along the negative real axis),

$$
L_{f}(x)=\ln \left(-\frac{m_{f}^{2}}{x+i \delta}\right), \quad \delta \rightarrow 0_{+} .
$$

\footnotetext{
2 Here, the argument $x$ of SE2 $12 \mathrm{~m}[\mathrm{x}]$ is one of the relativistic invariants $s, t, u$. This deviates from earlier conventions, where we denoted by $x$ the dimensionless conformal transform of $s, t, u$. This remark applies also to master integrals in Appendix A.
} 

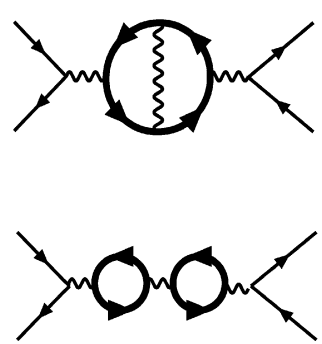

$2 b$

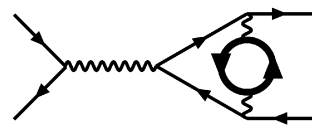

$2 d$
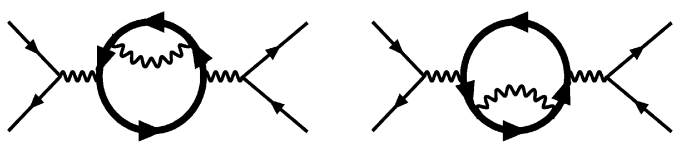

$2 \mathrm{a}$

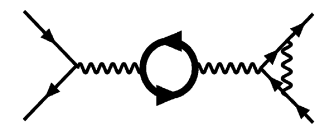

$2 \mathrm{c}$

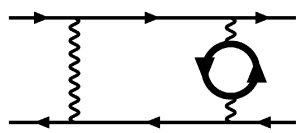

$2 \mathrm{e}$

Fig. 2. Classes of Bhabha-scattering two-loop diagrams containing at least one fermion loop. We use the conventions of Fig. 1. Note that class 2 a contains three topologically different subclasses. We refer to [26] for the reproduction of the full set of graphs.

Finally, neglecting $\mathcal{O}\left(m_{f}^{2} / x\right)$ terms, $\Pi_{f}^{(1)}(x)$ reads as

$$
\Pi_{f}^{(1)}(x)=-\frac{F_{\epsilon}}{3}\left(\frac{m_{e}^{2}}{m_{f}^{2}}\right)^{\epsilon}\left\{\frac{5}{3}+L_{f}(x)+\epsilon\left[\frac{28}{9}-\zeta_{2}+\frac{5}{3} L_{f}(x)+\frac{1}{2} L_{f}^{2}(x)\right]\right\} .
$$

Note that the $\mathcal{O}(\epsilon)$ term in Eq. (2.18) is not required for the NLO computation, but it will become relevant at NNLO. Here $\Pi_{f}^{(1)}(x)$ will be combined with infrared-divergent graphs showing single poles in the $\epsilon$ plane for $\epsilon=0$. The exact result for $\Pi_{f}^{(1)}(x)$ is available at [26].

\subsection{Outline of the NNLO computation}

At NNLO we have to consider:

- The interference of the two-loop diagrams of classes 2a-2e (see Fig. 2) with the tree-level amplitude;

- The interference of the one-loop vacuum-polarization diagrams of class 1a with the full set of graphs of classes 1a-1c (see Fig. 1).

The complete result can be organized as

$$
\frac{d \sigma^{\mathrm{NNLO}}}{d \Omega}=\underbrace{\sum_{\mathrm{i}=\mathrm{a}, \ldots, \mathrm{e}} \frac{d \sigma^{2 \mathrm{i} \times \text { tree }}}{d \Omega}}_{\text {2-loop } \times \text { tree }}+\underbrace{\sum_{\mathrm{i}=\mathrm{a}, \ldots, \mathrm{c}} \frac{d \sigma^{1 \mathrm{a} \times 1 \mathrm{i}}}{d \Omega}}_{1 \text {-loop } \times 1 \text {-loop }} .
$$

In order to compute the NNLO differential cross section we use the following reduction strategy:

- The generation of all the diagrams is simple and has been made with the computer-algebra systems GraphShot [27] and qgraf / DIANA [28-30]. We spin-sum the squared matrix 
elements and take the traces over Dirac indices in $D$ dimensions using the computer-algebra system FORM [31]. The resulting expressions are combinations of algebraic coefficients depending on $s, t, u, m_{e}, m_{f}$ and $\epsilon$ and two-loop integrals with scalar products containing the loop momenta in the numerators. An example showing the complexity of the result (two-loop box diagram of class 2e, see Fig. 2) can be found at [26].

- We reduce the loop integrals to a set of master integrals by means of the IdSolver implementation [32] of the Laporta algorithm [33,34]. The complete list of massive Bhabhascattering master integrals can be found in [22].

Next, we evaluate the master integrals:

- Integrals arising from graphs of classes 1a-1c (Fig. 1), 2a-2c (Fig. 2) and 2d-2e (Fig. 2, with electron loops) have been computed exactly through the method of differential equations in the external kinematic variables and expressed through harmonic polylogarithms [35] or generalized harmonic polylogarithms [36,37]. Here we agree perfectly with the work of [17, 25]. Non-approximated results for the various components of the differential cross section are collected in a Mathematica [38] file at [26].

- Integrals generated by the diagrams of classes $2 \mathrm{~d}-2 \mathrm{e}$ (Fig. 2, with heavy-fermion loops) are computed through a method based on asymptotic expansions of Mellin-Barnes representations. We derived appropriate Mellin-Barnes representations $[39,40]$ for each master integral and performed an analytic continuation in $\epsilon$ from a range where the integral is regular to the origin of the $\epsilon$ plane [4,5]. This is done by an automatic procedure implemented in the package MB.m [41]. To proceed further, we assume a hierarchy of scales, $m_{e}^{2} \ll m_{f}^{2} \ll s, t, u$, where $f \neq e$. After identifying the leading contributions in the fermion masses (in the same spirit as in [42]), we express the integrals by series over residua, and the latter are summed up analytically in terms of polylogs by means of the package XSUMMER [43]. Asymptotic expansions for the master integrals with two different masses were given in [44]. They, and also few lacking expansions of simpler masters needed here have been collected in Appendix A. We refer for a detailed discussion to [24], where the technique was employed to derive approximated results for the massive Bhabha-scattering planar box master integrals. All the mass-expanded masters may also be found in a Mathematica file at [26].

\subsection{Renormalization}

In the following we will always deal with ultraviolet-renormalized quantities. After regularizing the theory using dimensional regularization $[45,46]$, we perform renormalization in the on-mass-shell scheme. Here we relate all free parameters to physical observables:

- The electric charge coincides with the value of the electromagnetic coupling, as measured in Thomson scattering, at all orders in perturbation theory;

- The squared fermion masses are identified with the real parts of the poles of the Dysonresummed propagators;

- Finally, field-renormalization constants are chosen in order to cancel external wave-function corrections.

Counterterm-dependent Feynman rules are shown in Fig. 3. Note that the presence of infrared divergencies at NNLO requires to compute one-loop counterterms including $\mathcal{O}(\epsilon)$ terms. 


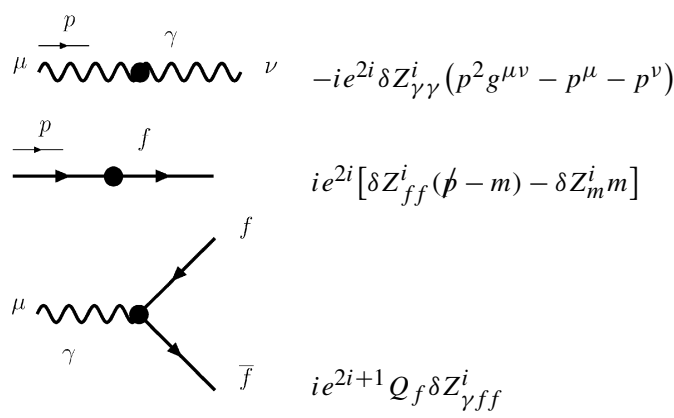

Fig. 3. Counterterm-dependent Feynman rules relevant for Bhabha scattering for $i=1$ (one loop) and $i=2$ (two loops). Note that in the on-mass-shell scheme $e^{2}=4 \pi \alpha$ at all orders in perturbation theory.

\subsubsection{One-loop counterterms}

The one-loop counterterms read as

$$
\begin{aligned}
& \delta Z_{\gamma \gamma}^{1}=-\frac{F_{\epsilon}}{12 \pi^{2}} \sum_{f} Q_{f}^{2}\left(\frac{m_{e}^{2}}{m_{f}^{2}}\right)^{\epsilon}\left(\frac{1}{\epsilon}+\frac{\epsilon}{2} \zeta_{2}\right), \\
& \delta Z_{f f}^{1}=\delta Z_{m}^{1}=-\frac{F_{\epsilon}}{16 \pi^{2}} Q_{f}^{2}\left(\frac{m_{e}^{2}}{m_{f}^{2}}\right)^{\epsilon}\left[\frac{3}{\epsilon}+4+\epsilon\left(8+\frac{3}{2} \zeta_{2}\right)\right], \\
& \delta Z_{\gamma f f}^{1}=\delta Z_{f f}^{1},
\end{aligned}
$$

where the last equation follows from the U(1) QED Ward identity. In the ultrarelativistic limit, the one-loop fermion-mass counterterm is not needed, since it is always multiplied by the fermion mass. Note however that the same counterterm is relevant for the exact computation.

\subsubsection{Two-loop counterterms}

At the two-loop level we get

$$
\begin{aligned}
& \delta Z_{\gamma \gamma}^{2}=-\frac{F_{\epsilon}^{2}}{128 \pi^{4}} \sum_{f} Q_{f}^{4}\left(\frac{m_{e}^{2}}{m_{f}^{2}}\right)^{2 \epsilon}\left(\frac{1}{\epsilon}+\frac{15}{2}\right) \\
& \delta Z_{\gamma e e}^{2}=\frac{F_{\epsilon}^{2}}{128 \pi^{4}}\left[\frac{1}{2 \epsilon}+\frac{947}{36}-16 \zeta_{2}+\sum_{f \neq e} Q_{f}^{2}\left(\frac{m_{e}^{2}}{m_{f}^{2}}\right)^{2 \epsilon}\left(\frac{1}{2 \epsilon}-\frac{5}{12}\right)\right] .
\end{aligned}
$$

The result for $\delta Z_{\gamma e e}^{2}$ is obtained including just fermion-loop diagrams and neglecting $\mathcal{O}\left(\mathrm{m}_{e}^{2} / \mathrm{m}_{f}^{2}\right)$ terms for $f \neq e$. The expression for $\delta Z_{\gamma \gamma}^{2}$ (as well as the one-loop counterterms of Eqs. (2.20)(2.22)), instead, is exact, since it follows from the single-scale diagrams of classes $2 \mathrm{a}-2 \mathrm{~b}$ of Fig. 2. Finally, we observe that the two-loop counterterm with two fermion lines is not required, since the use of an on-mass-shell renormalization removes external wave-function factors.

\section{Two-loop corrections}

In this section we show our approximated results for all the components of the NNLO differential cross section of Eq. (2.6). Our short-hand notation for logarithmic functions can be found 
in Eq. (2.17). In addition, we define two combinations of the Mandelstam invariants:

$$
\begin{aligned}
& v_{1}(x, y ; \epsilon)=x^{2}+2 y^{2}+2 x y-\epsilon x^{2}, \\
& v_{2}(x, y ; \epsilon)=(x+y)^{2}-\epsilon\left(x^{2}+y^{2}+x y\right),
\end{aligned}
$$

where $x(y)=s, t, u$. Note that for $\epsilon=0$ these functions are proportional to the kinematical factors appearing in the Born cross section of Eq. (2.5) and the NLO corrections of Eq. (2.7). Moreover, we introduce a compact notation which will prove useful in discussing box corrections in Section 3.3 and the complete NNLO differential cross section in Section 4,

$$
L\left(R_{f}\right)=\ln \left(\frac{m_{e}^{2}}{m_{f}^{2}}\right) .
$$

\subsection{Vacuum-polarization corrections}

The interference of the vacuum-polarization diagrams of classes $2 \mathrm{a}$ and $2 \mathrm{~b}$ with the tree-level amplitude can be written as

$$
\begin{aligned}
\frac{d \sigma^{2 \mathrm{i} \times \text { tree }}}{d \Omega}= & \frac{\alpha^{2}}{s}\left\{\frac{1}{s^{2}} v_{1}(s, t ; 0) A^{2 \mathrm{i}}(s)+\frac{1}{t^{2}} v_{1}(t, s ; 0) A^{2 \mathrm{i}}(t)\right. \\
& \left.+\frac{1}{s t} v_{2}(s, t ; 0)\left[A^{2 \mathrm{i}}(s)+A^{2 \mathrm{i}}(t)\right]\right\}, \quad \mathrm{i}=\mathrm{a}, \mathrm{b} .
\end{aligned}
$$

Here we introduced the auxiliary functions $A^{2 \mathrm{a}}(x)$ and $A^{2 \mathrm{~b}}(x)$, which are expressed through the renormalized one- and two-loop vacuum-polarization functions $\Pi_{f}^{(1)}(x)$ (see Eq. (2.18)) and $\Pi_{f}^{(2)}(x)$,

$$
\begin{aligned}
& A^{2 \mathrm{a}}(x)=\sum_{f} Q_{f}^{4} \operatorname{Re}\left[\Pi_{f}^{(2)}(x)\right], \\
& A^{2 \mathrm{~b}}(x)=\sum_{f_{1}, f_{2}} Q_{f_{1}}^{2} Q_{f_{2}}^{2} \operatorname{Re}\left[\Pi_{f_{1}}^{(1)}(x) \Pi_{f_{2}}^{(1)}(x)\right],
\end{aligned}
$$

where the result for $\Pi_{f}^{(2)}(x)$ in the small fermion-mass limit reads as

$$
\Pi_{f}^{(2)}(x)=-\frac{5}{24}+\zeta_{3}-\frac{1}{4} L_{f}(x) .
$$

Note that $\mathcal{O}(\epsilon)$ terms in Eq. (3.4) coming from the kinematical coefficients of Eq. (3.1) can be safely neglected, since both $\Pi_{f}^{(1)}(x)$ and $\Pi_{f}^{(2)}(x)$ are infrared-finite quantities.

\subsection{Vertex corrections}

The contribution of reducible (irreducible) vertex corrections to the NNLO differential cross section can be readily derived from diagrams of classes $2 \mathrm{c}(2 \mathrm{~d})$ in Fig. 2,

$$
\begin{aligned}
\frac{d \sigma^{2 \mathrm{i} \times \text { tree }}}{d \Omega}= & 2 \frac{\alpha^{2}}{s}\left\{\frac{1}{s^{2}}\left[v_{1}(s, t ; \epsilon) A_{\mathrm{V}}^{2 \mathrm{i}}(s)+s^{2} A_{\mathrm{M}}^{2 \mathrm{i}}(s)\right]+\frac{1}{t^{2}}\left[v_{1}(t, s ; \epsilon) A_{\mathrm{V}}^{2 \mathrm{i}}(t)+t^{2} A_{\mathrm{M}}^{2 \mathrm{i}}(t)\right]\right. \\
& +\frac{1}{s t}\left[v_{2}(s, t ; \epsilon)\left(A_{\mathrm{V}}^{2 \mathrm{i}}(s)+A_{\mathrm{V}}^{2 \mathrm{i}}(t)\right)+\frac{3}{2}\left(s^{2} A_{\mathrm{M}}^{2 \mathrm{i}}(s)+t^{2} A_{\mathrm{M}}^{2 \mathrm{i}}(t)\right)\right. \\
& \left.\left.+2 s t\left(A_{\mathrm{M}}^{2 \mathrm{i}}(s)+A_{\mathrm{M}}^{2 \mathrm{i}}(t)\right)\right]\right\}, \quad \mathrm{i}=\mathrm{c}, \mathrm{d} .
\end{aligned}
$$




\subsubsection{Reducible diagrams}

The auxiliary functions $A_{\mathrm{V}}^{2 \mathrm{c}}(x)$ and $A_{\mathrm{M}}^{2 \mathrm{c}}(x)$ are given by the product of the renormalized oneloop vacuum-polarization function $\Pi_{f}^{(1)}(x)$ (expanded in Eq. (2.18) including $\mathcal{O}(\epsilon)$ terms) and the renormalized one-loop vector and magnetic vertex form factors $F_{\mathrm{V}}^{(1)}(x)$ and $F_{\mathrm{M}}^{(1)}(x)$,

$$
A_{\mathrm{I}}^{2 \mathrm{c}}(x)=\sum_{f} Q_{f}^{2} \operatorname{Re}\left[F_{\mathrm{I}}^{(1)}(x) \Pi_{f}^{(1)}(x)\right], \quad \mathrm{I}=\mathrm{V}, \mathrm{M} .
$$

The asymptotic expansion of $F_{\mathrm{V}}^{(1)}(x)$ is given by

$$
F_{\mathrm{V}}^{1}(x)=-\frac{F_{\epsilon}}{2 \epsilon}\left[1+L_{e}(x)\right]-1+\frac{1}{2} \zeta_{2}-\frac{3}{4} L_{e}(x)-\frac{1}{4} L_{e}^{2}(x),
$$

whereas $F_{\mathrm{M}}^{(1)}(x)$ vanishes when we neglect the electron mass, $F_{\mathrm{M}}^{(1)}(x)=0$. The renormalized one-loop vertex develops an infrared divergency, which shows up as a single pole in the $\epsilon$ plane for $\epsilon=0$. Therefore, when computing the cross section, we sum over the spins the squared matrix element and we evaluate the traces over Dirac indices in $D=4-2 \epsilon$ dimensions. The needed kinematical structures include $\mathcal{O}(\epsilon)$ terms (see Eq. (3.1)).

\subsubsection{Irreducible diagrams}

The renormalized two-loop vertex diagrams of class $2 \mathrm{~d}$ are free of infrared divergencies. Therefore, we can neglect $\mathcal{O}(\epsilon)$ terms in the kinematical coefficients of Eq. (3.1) appearing in Eq. (3.8), setting $v_{a}(x, y ; \epsilon)=v_{a}(x, y ; 0)$, for $a=1,2$. The auxiliary functions $A_{\mathrm{V}}^{2 \mathrm{~d}}(x)$ and $A_{\mathrm{M}}^{2 \mathrm{~d}}(x)$ contain the renormalized two-loop vector and magnetic vertex form factors (see [47-49] for a detailed discussion),

$$
A_{\mathrm{I}}^{2 \mathrm{~d}}(x)=\sum_{f} Q_{f}^{2} \operatorname{Re}\left[F_{\mathrm{I}, f}^{(2)}(x)\right], \quad \mathrm{I}=\mathrm{V}, \mathrm{M} .
$$

For the case with an electron loop, $F_{\mathrm{I}, e}^{(2)}(x)$, the exact results in terms of harmonic polylogarithms, can be readily expanded in the high-energy limit. For the vector term we get

$$
F_{\mathrm{V}, e}^{(2)}(x)=\frac{1}{4}\left(\frac{383}{27}-\zeta_{2}\right)+\frac{1}{6}\left(\frac{265}{36}+\zeta_{2}\right) L_{e}(x)+\frac{19}{72} L_{e}^{2}(x)+\frac{1}{36} L_{e}^{3}(x) .
$$

For $F_{\mathrm{V}, f}^{(2)}(x), f \neq e$, we perform an asymptotic expansion of the master integrals arising in the computation (see Table $\mathrm{V}$ in [22]) and we fully agree with the result of [50],

$$
\begin{aligned}
F_{\mathrm{V}, f}^{(2)}(x)= & \frac{1}{6}\left(\frac{3355}{216}+\frac{19}{6} \zeta_{2}-2 \zeta_{3}\right)+\frac{1}{6}\left(\frac{265}{36}+\zeta_{2}\right) L_{f}(x) \\
& +\frac{19}{72} L_{f}^{2}(x)+\frac{1}{36} L_{f}^{3}(x) .
\end{aligned}
$$

Since collinear logarithms are absent, the logarithmic structure of Eqs. (3.12) and (3.13) is obviously the same.

\subsection{Box corrections}

The contribution of the renormalized two-loop box diagrams of class $2 \mathrm{e}$ is given by

$$
\frac{d \sigma^{2 \mathrm{e} \times \text { tree }}}{d \Omega}=\frac{\alpha^{2}}{2 s}\left[\frac{1}{s} A_{1}^{2 \mathrm{e} \times \text { tree }}(s, t)+\frac{1}{t} A_{2}^{2 \mathrm{e} \times \text { tree }}(s, t)\right] .
$$


Here the auxiliary functions can be conveniently expressed through three independent form factors $B_{\mathrm{I}, f}^{(2)}(x, y)$, where $\mathrm{i}=\mathrm{A}, \mathrm{B}, \mathrm{C}$,

$$
\begin{aligned}
& A_{1}^{2 \mathrm{e} \times \operatorname{tree}}(s, t) \\
& \quad=F_{\epsilon}^{2} \sum_{f} Q_{f}^{2} \operatorname{Re}\left[B_{\mathrm{A}, f}^{(2)}(s, t)+B_{\mathrm{B}, f}^{(2)}(t, s)+B_{\mathrm{C}, f}^{(2)}(u, t)-B_{\mathrm{B}, f}^{(2)}(u, s)\right], \\
& A_{2}^{2 \mathrm{e} \times \operatorname{tree}}(s, t) \\
& \quad=F_{\epsilon}^{2} \sum_{f} Q_{f}^{2} \operatorname{Re}\left[B_{\mathrm{B}, f}^{(2)}(s, t)+B_{\mathrm{A}, f}^{(2)}(t, s)-B_{\mathrm{B}, f}^{(2)}(u, t)+B_{\mathrm{C}, f}^{(2)}(u, s)\right] .
\end{aligned}
$$

\subsubsection{Electron loops}

For the case with an electron loop, $B_{\mathrm{I}, e}^{(2)}(x, y)$, we get exact results in terms of harmonic polylogarithms and generalized harmonic polylogarithms. An asymptotic expansion in the limit $m_{e}^{2} \ll s, t, u$ leads to

$$
\begin{aligned}
& B_{\mathrm{A}, e}^{(2)}(x, y)=\frac{1}{\epsilon} \frac{2}{3}\left(\frac{x^{2}}{y}+2 x+y\right)\left[\frac{5}{3}+L_{e}(y)\right] L_{e}(x)+\frac{1}{3} \frac{x^{2}}{y}\left\{-\frac{2}{3}\left(\frac{17}{3}+20 \zeta_{2}\right)\right. \\
& +2\left(\frac{41}{9}-\zeta_{2}\right) L_{e}(x)-2\left(\frac{1}{3}+8 \zeta_{2}\right) L_{e}(y)-\frac{23}{6} L_{e}^{2}(y)+8 L_{e}(x) L_{e}(y) \\
& -\frac{5}{3} L_{e}^{3}(y)+4 L_{e}(x) L_{e}^{2}(y)-\left[6 \zeta_{2}+\ln ^{2}\left(\frac{y}{x}\right)\right] \ln \left(1+\frac{y}{x}\right) \\
& \left.-2 \ln \left(\frac{y}{x}\right) \operatorname{Li}_{2}\left(-\frac{y}{x}\right)+2 \operatorname{Li}_{3}\left(-\frac{y}{x}\right)\right\}+\frac{x}{3}\left\{-\frac{2}{3}\left(\frac{34}{3}+7 \zeta_{2}\right)+\frac{242}{9} L_{e}(x)\right. \\
& -4\left(\frac{5}{3}+6 \zeta_{2}\right) L_{e}(y)+\frac{1}{3}\left[13 L_{e}^{2}(x)-16 L_{e}^{2}(y)+34 L_{e}(x) L_{e}(y)\right] \\
& +2\left[\frac{1}{3} L_{e}^{3}(x)-L_{e}^{3}(y)+3 L_{e}(x) L_{e}^{2}(y)\right]-2\left[6 \zeta_{2}+\ln ^{2}\left(\frac{y}{x}\right)\right] \ln \left(1+\frac{y}{x}\right) \\
& \left.-4 \ln \left(\frac{y}{x}\right) \operatorname{Li}_{2}\left(-\frac{y}{x}\right)+4 \operatorname{Li}_{3}\left(-\frac{y}{x}\right)\right\}+\frac{y}{3}\left\{-\frac{2}{3}\left(\frac{17}{3}+11 \zeta_{2}\right)+\frac{130}{9} L_{e}(x)\right. \\
& -6\left(1+2 \zeta_{2}\right) L_{e}(y)+\frac{5}{3}\left[L_{e}^{2}(x)-\frac{5}{2} L_{e}^{2}(y)+4 L_{e}(x) L_{e}(y)\right]+\frac{1}{3} L_{e}^{3}(x) \\
& +3 L_{e}(x) L_{e}^{2}(y)-L_{e}^{3}(y)-\left[6 \zeta_{2}+\ln ^{2}\left(\frac{y}{x}\right)\right] \ln \left(1+\frac{y}{x}\right) \\
& \left.-2 \ln \left(\frac{y}{x}\right) \operatorname{Li}_{2}\left(-\frac{y}{x}\right)+2 \operatorname{Li}_{3}\left(-\frac{y}{x}\right)\right\}, \\
& B_{\mathrm{B}, e}^{(2)}(x, y)=\frac{1}{\epsilon} \frac{2}{3}\left(2 \frac{x^{2}}{y}+2 x+y\right)\left[\frac{5}{3}+L_{e}(y)\right] L_{e}(x)+\frac{1}{3} \frac{x^{2}}{y}\left\{\frac{4}{3}\left(-\frac{17}{3}-20 \zeta_{2}\right)\right. \\
& +4\left(\frac{56}{9}-\zeta_{2}\right) L_{e}(x)-4\left(\frac{1}{3}+8 \zeta_{2}\right) L_{e}(y)-\left[\frac{23}{3} L_{e}^{2}(y)-20 L_{e}(x) L_{e}(y)\right] \\
& -2\left[\frac{5}{3} L_{e}^{3}(y)-4 L_{e}(x) L_{e}^{2}(y)\right]-2\left[6 \zeta_{2}+\ln ^{2}\left(\frac{y}{x}\right)\right] \ln \left(1+\frac{y}{x}\right)
\end{aligned}
$$




$$
\begin{aligned}
& \left.-4 \ln \left(\frac{y}{x}\right) \operatorname{Li}_{2}\left(-\frac{y}{x}\right)+4 \operatorname{Li}_{3}\left(-\frac{y}{x}\right)\right\}+\frac{x}{3}\left\{-\frac{2}{3}\left(\frac{34}{3}+7 \zeta_{2}\right)+\frac{272}{9} L_{e}(x)\right. \\
& -4\left(\frac{5}{3}+6 \zeta_{2}\right) L_{e}(y)+\frac{1}{3}\left[13 L_{e}^{2}(x)+40 L_{e}(x) L_{e}(y)-16 L_{e}^{2}(y)\right] \\
& +2\left[\frac{1}{3} L_{e}^{3}(x)-L_{e}^{3}(y)+3 L_{e}(x) L_{e}^{2}(y)\right]-2\left[6 \zeta_{2}+\ln ^{2}\left(\frac{y}{x}\right)\right] \ln \left(1+\frac{y}{x}\right) \\
& \left.-4 \ln \left(\frac{y}{x}\right) \operatorname{Li}_{2}\left(-\frac{y}{x}\right)+4 \operatorname{Li}_{3}\left(-\frac{y}{x}\right)\right\}+\frac{y}{3}\left\{-\frac{2}{3}\left(\frac{17}{3}+11 \zeta_{2}\right)+\frac{130}{9} L_{e}(x)\right. \\
& -6\left(1+2 \zeta_{2}\right) L_{e}(y)+\frac{5}{3}\left[L_{e}^{2}(x)-\frac{5}{2} L_{e}^{2}(y)+4 L_{e}(x) L_{e}(y)\right]+\frac{1}{3} L_{e}^{3}(x) \\
& -L_{e}^{3}(y)+3 L_{e}(x) L_{e}^{2}(y)-\left[6 \zeta_{2}+\ln ^{2}\left(\frac{y}{x}\right)\right] \ln \left(1+\frac{y}{x}\right) \\
& \left.-2 \ln \left(\frac{y}{x}\right) \mathrm{Li}_{2}\left(-\frac{y}{x}\right)+2 \mathrm{Li}_{3}\left(-\frac{y}{x}\right)\right\} \text {, } \\
& B_{\mathrm{C}, e}^{(2)}(x, y)=-\frac{1}{\epsilon} \frac{2}{3} \frac{x^{2}}{y}\left[\frac{5}{3}+L_{e}(y)\right] L_{e}(x)+\frac{2}{3}(x+y)\left[\frac{5}{3}+L_{e}(y)\right] L_{e}(x) \\
& +\frac{1}{3} \frac{x^{2}}{y}\left\{\frac{2}{3}\left(\frac{17}{3}+20 \zeta_{2}\right)-2\left(\frac{41}{9}-\zeta_{2}\right) L_{e}(x)+2\left(\frac{1}{3}+8 \zeta_{2}\right) L_{e}(y)\right. \\
& +\frac{23}{6} L_{e}^{2}(y)-8 L_{e}(x) L_{e}(y)+\frac{5}{3} L_{e}^{3}(y)-4 L_{e}(x) L_{e}^{2}(y) \\
& \left.+\left[6 \zeta_{2}+\ln ^{2}\left(\frac{y}{x}\right)\right] \ln \left(1+\frac{y}{x}\right)+2 \ln \left(\frac{y}{x}\right) \operatorname{Li}_{2}\left(-\frac{y}{x}\right)-2 \operatorname{Li}_{3}\left(-\frac{y}{x}\right)\right\} .
\end{aligned}
$$

\subsubsection{Heavy-fermion loops}

The list of master integrals used here was given in Table V of [22]. In Appendix A we collect the explicit analytic expressions for them in the ultra-relativistic limit. At variance with the electron-loop case, it is not possible to compute them exactly by means of a basis containing harmonic polylogarithms and generalized harmonic polylogarithms. Therefore, we use the highenergy asymptotic expansion discussed in Section 2.2. The results, expressed by the logarithms of the fermion masses $L\left(R_{f}\right)$ (see Eq. (3.3)), are:

$$
\begin{aligned}
B_{\mathrm{A}, f}^{(2)}(x, y)= & \frac{1}{\epsilon} \frac{2}{3}\left(\frac{x^{2}}{y}+2 x+y\right)\left[\frac{5}{3}-L\left(R_{f}\right)+L_{e}(y)\right] L_{e}(x) \\
& +\frac{1}{3} \frac{x^{2}}{y}\left\{2\left(\frac{131}{27}-10 \zeta_{2}-2 \zeta_{3}\right)-2\left(\frac{25}{9}-6 \zeta_{2}\right) L\left(R_{f}\right)\right. \\
& +\frac{7}{6} L^{2}\left(R_{f}\right)-\frac{1}{3} L^{3}\left(R_{f}\right)+\left[\frac{82}{9}-2 \zeta_{2}-\frac{4}{3} L\left(R_{f}\right)\right] L_{e}(x) \\
& -2\left[\frac{1}{3}+8 \zeta_{2}-\frac{1}{2} L\left(R_{f}\right)\right] L_{e}(y)-\left[\frac{23}{6}-2 L\left(R_{f}\right)\right] L_{e}^{2}(y) \\
& +4\left[2-L\left(R_{f}\right)\right] L_{e}(x) L_{e}(y)-4\left[\frac{5}{12} L_{e}^{3}(y)-L_{e}(x) L_{e}^{2}(y)\right]
\end{aligned}
$$




$$
\begin{aligned}
& \left.-\left[6 \zeta_{2}+\ln ^{2}\left(\frac{y}{x}\right)\right] \ln \left(1+\frac{y}{x}\right)-2 \ln \left(\frac{y}{x}\right) \operatorname{Li}_{2}\left(-\frac{y}{x}\right)-2 \operatorname{Li}_{3}\left(-\frac{y}{x}\right)\right\} \\
& +\frac{x}{3}\left\{2\left(\frac{262}{27}-9 \zeta_{2}-4 \zeta_{3}\right)-4\left(\frac{25}{9}-3 \zeta_{2}\right) L\left(R_{f}\right)\right. \\
& +\frac{7}{3} L^{2}\left(R_{f}\right)-\frac{2}{3} L^{3}\left(R_{f}\right)+2\left[\frac{121}{9}-\frac{10}{3} L\left(R_{f}\right)\right] L_{e}(x)-2\left[\frac{10}{3}+12 \zeta_{2}\right. \\
& \left.-2 L\left(R_{f}\right)\right] L_{e}(y)+\left[\frac{13}{3}-2 L\left(R_{f}\right)\right] L_{e}^{2}(x)-\left[\frac{16}{3}-2 L\left(R_{f}\right)\right] L_{e}^{2}(y) \\
& +2\left[\frac{17}{3}-2 L\left(R_{f}\right)\right] L_{e}(x) L_{e}(y)+\frac{2}{3} L_{e}^{3}(x)+6 L_{e}(x) L_{e}^{2}(y)-2 L_{e}^{3}(y) \\
& \left.-2\left[6 \zeta_{2}+\ln ^{2}\left(\frac{y}{x}\right)\right] \ln \left(1+\frac{y}{x}\right)-4 \ln \left(\frac{y}{x}\right) \operatorname{Li}_{2}\left(-\frac{y}{x}\right)+4 \operatorname{Li}_{3}\left(-\frac{y}{x}\right)\right\} \\
& +\frac{y}{3}\left\{2\left(\frac{131}{27}-7 \zeta_{2}-2 \zeta_{3}\right)-2\left(\frac{25}{9}-3 \zeta_{2}\right) L\left(R_{f}\right)+\frac{7}{6} L^{2}\left(R_{f}\right)\right. \\
& -\frac{1}{3} L^{3}\left(R_{f}\right)+\left[\frac{130}{9}-\frac{10}{3} L\left(R_{f}\right)\right] L_{e}(x)-\left[6+12 \zeta_{2}-3 L\left(R_{f}\right)\right] L_{e}(y) \\
& +\left[\frac{5}{3}-L\left(R_{f}\right)\right] L_{e}^{2}(x)-\left[\frac{25}{6}-L\left(R_{f}\right)\right] L_{e}^{2}(y) \\
& +2\left[\frac{10}{3}-L\left(R_{f}\right)\right] L_{e}(x) L_{e}(y)+\frac{1}{3} L_{e}^{3}(x)-L_{e}^{3}(y)+3 L_{e}(x) L_{e}^{2}(y) \\
& \left.-\left[6 \zeta_{2}+\ln ^{2}\left(\frac{y}{x}\right)\right] \ln \left(1+\frac{y}{x}\right)-2 \ln \left(\frac{y}{x}\right) \operatorname{Li}_{2}\left(-\frac{y}{x}\right)+2 \operatorname{Li}_{3}\left(-\frac{y}{x}\right)\right\}
\end{aligned}
$$$$
B_{\mathrm{B}, f}^{(2)}(x, y)=\frac{1}{\epsilon} \frac{2}{3}\left(2 \frac{x^{2}}{y}+2 x+y\right)\left[\frac{5}{3}-L\left(R_{f}\right)+L_{e}(y)\right] L_{e}(x)
$$$$
+\frac{2}{3} \frac{x^{2}}{y}\left\{\frac{262}{27}-20 \zeta_{2}-4 \zeta_{3}-\left(\frac{50}{9}-12 \zeta_{2}\right) L\left(R_{f}\right)+\frac{7}{6} L^{2}\left(R_{f}\right)\right.
$$$$
-\frac{1}{3} L^{3}\left(R_{f}\right)+\left[\frac{112}{9}-2 \zeta_{2}-\frac{10}{3} L\left(R_{f}\right)\right] L_{e}(x)+\left[-\frac{2}{3}-16 \zeta_{2}\right.
$$$$
\left.+L\left(R_{f}\right)\right] L_{e}(y)-\left[\frac{23}{6}-2 L\left(R_{f}\right)\right] L_{e}^{2}(y)+2\left[5-2 L\left(R_{f}\right)\right] L_{e}(x) L_{e}(y)
$$$$
-4\left[\frac{5}{12} L_{e}^{3}(y)-L_{e}(x) L_{e}^{2}(y)\right]-\left[6 \zeta_{2}+\ln ^{2}\left(\frac{y}{x}\right)\right] \ln \left(1+\frac{y}{x}\right)
$$$$
\left.-2 \ln \left(\frac{y}{x}\right) \mathrm{Li}_{2}\left(-\frac{y}{x}\right)+2 \mathrm{Li}_{3}\left(-\frac{y}{x}\right)\right\}
$$$$
+\frac{2 x}{3}\left\{\frac{262}{27}-9 \zeta_{2}-4 \zeta_{3}-2\left(\frac{25}{9}-3 \zeta_{2}\right) L\left(R_{f}\right)+\frac{7}{6} L^{2}\left(R_{f}\right)\right.
$$$$
-\frac{1}{3} L^{3}\left(R_{f}\right)+\left[\frac{136}{9}-\frac{13}{3} L\left(R_{f}\right)\right] L_{e}(x)-\left[\frac{10}{3}+12 \zeta_{2}-2 L\left(R_{f}\right)\right] L_{e}(y)
$$$$
+\left[\frac{13}{6}-L\left(R_{f}\right)\right] L_{e}^{2}(x)-\left[\frac{8}{3}-L\left(R_{f}\right)\right] L_{e}^{2}(y)
$$ 


$$
\begin{aligned}
& +\left[\frac{20}{3}-2 L\left(R_{f}\right)\right] L_{e}(x) L_{e}(y) \\
& +\frac{1}{3} L_{e}^{3}(x)+3 L_{e}(x) L_{e}^{2}(y)-L_{e}^{3}(y)-\left[6 \zeta_{2}+\ln ^{2}\left(\frac{y}{x}\right)\right] \ln \left(1+\frac{y}{x}\right) \\
& \left.-2 \ln \left(\frac{y}{x}\right) \operatorname{Li}_{2}\left(-\frac{y}{x}\right)+2 \operatorname{Li}_{3}\left(-\frac{y}{x}\right)\right\}+\frac{2 y}{3}\left\{\left(\frac{131}{27}-7 \zeta_{2}-2 \zeta_{3}\right)\right. \\
& -\left(\frac{25}{9}-3 \zeta_{2}\right) L\left(R_{f}\right)+\frac{7}{12} L^{2}\left(R_{f}\right)-\frac{1}{6} L^{3}\left(R_{f}\right)+\left[\frac{65}{9}-\frac{5}{3} L\left(R_{f}\right)\right] L_{e}(x) \\
& -\frac{1}{2}\left[6+12 \zeta_{2}-3 L\left(R_{f}\right)\right] L_{e}(y)+\frac{1}{2}\left[\frac{5}{3}-L\left(R_{f}\right)\right] L_{e}^{2}(x) \\
& -\frac{1}{2}\left[\frac{25}{6}-L\left(R_{f}\right)\right] L_{e}^{2}(y)+\left[\frac{10}{3}-L\left(R_{f}\right)\right] L_{e}(x) L_{e}(y)+\frac{1}{6} L_{e}^{3}(x) \\
& -\frac{1}{2} L_{e}^{3}(y)+\frac{3}{2} L_{e}(x) L_{e}^{2}(y)-\left[3 \zeta_{2}+\frac{1}{2} \ln ^{2}\left(\frac{y}{x}\right)\right] \ln \left(1+\frac{y}{x}\right) \\
& \left.-\ln \left(\frac{y}{x}\right) \operatorname{Li}_{2}\left(-\frac{y}{x}\right)+\operatorname{Li}_{3}\left(-\frac{y}{x}\right)\right\}, \\
& B_{\mathrm{C}, f}^{(2)}(x, y)=-\frac{1}{\epsilon} \frac{2}{3} \frac{x^{2}}{y}\left[\frac{5}{3}-L\left(R_{f}\right)+L_{e}(y)\right] L_{e}(x)+\frac{2}{3}(x+y)\left[\frac{5}{3}-L\left(R_{f}\right)\right. \\
& \left.+L_{e}(y)\right] L_{e}(x)+\frac{2}{3} \frac{x^{2}}{y}\left\{-\frac{131}{27}+10 \zeta_{2}+2 \zeta_{3}+\left(\frac{25}{9}-6 \zeta_{2}\right) L\left(R_{f}\right)\right. \\
& -\frac{7}{12} L^{2}\left(R_{f}\right)+\frac{1}{6} L^{3}\left(R_{f}\right)-\left[\frac{41}{9}-\zeta_{2}-\frac{2}{3} L\left(R_{f}\right)\right] L_{e}(x)+\left[\frac{1}{3}+8 \zeta_{2}\right. \\
& \left.-\frac{1}{2} L\left(R_{f}\right)\right] L_{e}(y)-2\left[2-L\left(R_{f}\right)\right] L_{e}(x) L_{e}(y)+\left[\frac{23}{12}-L\left(R_{f}\right)\right] L_{e}^{2}(y) \\
& +\frac{5}{6} L_{e}^{3}(y)-2 L_{e}(x) L_{e}^{2}(y)+\left[3 \zeta_{2}+\frac{1}{2} \ln ^{2}\left(\frac{y}{x}\right)\right] \ln \left(1+\frac{y}{x}\right) \\
& \left.+\ln \left(\frac{y}{x}\right) \operatorname{Li}_{2}\left(-\frac{y}{x}\right)-\operatorname{Li}_{3}\left(-\frac{y}{x}\right)\right\} .
\end{aligned}
$$

In order to study the numerical effects of massive leptons in two-loop box diagrams we consider the interference of the box diagram of class 2e (see Fig. 2) with the s-channel tree-level amplitude,

$$
B_{2 \mathrm{e}, f}=\frac{\alpha^{2}}{4 s^{2}} \operatorname{Re}\left[B_{\mathrm{A}, f}^{(2)}(s, t)\right],
$$

where $B_{\mathrm{A}, f}$ can be found in Eq. (3.17) for electron loops, and in Eq. (3.20) for $f \neq e$ loops. In Table 1 (Table 2) we show numerical values for the finite part of $B_{2 \mathrm{e}, f}$ at values of $\sqrt{s}$ typical for meson factories, Giga-Z, ILC, and at two selected small and wide scattering angles, $\theta=3^{\circ}$ $\left(\theta=90^{\circ}\right)$.

For comparison we show in Table 3 the real part of the vertex function, see Eq. (3.13). We notice that the contributions of the box diagrams with heavier fermions are not strongly suppressed, and are comparable to those coming from the electron-loop boxes. This is different with respect 
Table 1

Numerical values for the finite part of $B_{2 \mathrm{e}, f}$ of Eq. (3.23) in nanobarns at a scattering angle $\theta=3^{\circ}$. The first two entries for the $\tau$ lepton are not shown since here the highenergy approximation in not justified (the same consideration applies to the top quark)

\begin{tabular}{llll}
\hline$B_{2 \mathrm{e}, f}[\mathrm{nb}] / \sqrt{s}[\mathrm{GeV}]$ & 10 & 91 & 500 \\
\hline$e$ [see Eq. (3.17)] & 188758 & 5200.08 & 284.711 \\
$\mu$ [see Eq. (3.20)] & 1635.62 & 1686.88 & 130.579 \\
$\tau$ [see Eq. (3.20)] & & & 39.5554 \\
\hline
\end{tabular}

Table 2

Numerical values for the finite part of $B_{2 \mathrm{e}, f}$ of Eq. (3.23) in nanobarns at a scattering angle $\theta=90^{\circ}$. The first two entries for the top quark are not shown since here the highenergy approximation in not justified

\begin{tabular}{lclc}
\hline$B_{2 \mathrm{e}, f}[\mathrm{nb}] / \sqrt{s}[\mathrm{GeV}]$ & 10 & 91 & 500 \\
\hline$e$ [see Eq. (3.17)] & 143.162 & 3.23102 & 0.160582 \\
$\mu$ [see Eq. (3.20)] & 61.3875 & 1.79381 & 0.0995184 \\
$\tau$ [see Eq. (3.20)] & 10.0105 & 0.935319 & 0.0639576 \\
$t$ [see Eq. (3.20)] & & & -0.00256757 \\
\hline
\end{tabular}

Table 3

The real part for the vertex form factor, see Eqs. (3.12) and (3.13)

\begin{tabular}{llll}
\hline$\sqrt{s}[\mathrm{GeV}]$ & 10 & 91 & 500 \\
\hline$e$ & -124.237 & -254.293 & -400.574 \\
$\mu$ & -4.8036 & -29.1057 & -70.1032 \\
$\tau$ & & -2.08719 & -13.4901 \\
\hline
\end{tabular}

to the self-energy and vertex corrections and may be traced back to the logarithmic structure of the terms in Eqs. (3.20)-(3.22), where terms of order $L_{e}^{3}(x)$ appear (note that the two-loop box master integrals of Eqs. (A.7) and (A.8) of Appendix A show a dependence on $L_{e}^{3}(x)$, in contrast to the vertex and self-energy masters with heavy fermion loops). After assembling the box diagrams we see a remaining dependence on $\ln ^{2}\left(s / m_{e}^{2}\right)$. This is a collinear mass singularity, coming from the external legs of the diagrams, which leads to the fact that the two-loop box corrections from heavier fermions are not numerically suppressed compared to the electron-loop contributions. One may control this easily by evaluating the singularity structure of the corresponding massless box diagram where only a scale $M$ due to the internal loop exists, and see there some $1 / \epsilon^{2}$ terms which are absent in the corresponding self-energy and vertex diagrams.

\subsection{Products of one-loop corrections}

Finally, we consider the simpler components generated by the interference of one-loop diagrams among themselves. We start with the interference of diagrams of class 1a,

$$
\begin{aligned}
\frac{d \sigma^{1 \mathrm{a} \times 1 \mathrm{a}}}{d \Omega}= & \frac{\alpha^{2}}{2 s}\left\{\frac{1}{s^{2}} v_{1}(s, t ; 0) A^{1 \mathrm{a} \times 1 \mathrm{a}}(s, s)+\frac{1}{t^{2}} v_{1}(t, s ; 0) A^{1 \mathrm{a} \times 1 \mathrm{a}}(t, t)\right. \\
& \left.+\frac{1}{s t} v_{2}(s, t ; 0)\left[A^{1 \mathrm{a} \times 1 \mathrm{a}}(s, t)+A^{1 \mathrm{a} \times 1 \mathrm{a}}(t, s)\right]\right\} .
\end{aligned}
$$


Here the auxiliary function $A^{1 \mathrm{a} \times 1 \mathrm{a}}(x, y)$ contains the product of the renormalized one-loop vacuum-polarization function $\Pi_{f}^{(1)}(x)$ (see Eq. (2.18)) with its complex conjugate,

$$
A^{1 \mathrm{a} \times 1 \mathrm{a}}(x, y) \equiv \sum_{f_{1}, f_{2}} Q_{f_{1}}^{2} Q_{f_{2}}^{2} \Pi_{f_{1}}^{(1)}(x)\left[\Pi_{f_{2}}^{(1)}(y)\right]^{\star} .
$$

The interference of diagrams of class $1 \mathrm{a}$ with those of class $1 \mathrm{~b}$ gives

$$
\begin{aligned}
\frac{d \sigma^{1 \mathrm{a} \times 1 \mathrm{~b}}}{d \Omega}= & 2 \frac{\alpha^{2}}{s}\left\{\frac{1}{s^{2}}\left[v_{1}(s, t ; \epsilon) A_{\mathrm{V}}^{1 \mathrm{a} \times 1 \mathrm{~b}}(s, s)+s^{2} A_{\mathrm{M}}^{1 \mathrm{a} \times 1 \mathrm{~b}}(s, s)\right]\right. \\
& +\frac{1}{t^{2}}\left[v_{1}(t, s ; \epsilon) A_{\mathrm{V}}^{1 \mathrm{a} \times 1 \mathrm{~b}}(t, t)+t^{2} A_{\mathrm{M}}^{1 \mathrm{a} \times 1 \mathrm{~b}}(t, t)\right] \\
& +\frac{1}{s t}\left[v_{2}(s, t ; \epsilon)\left(A_{\mathrm{V}}^{1 \mathrm{a} \times 1 \mathrm{~b}}(s, t)+A_{\mathrm{V}}^{1 \mathrm{a} \times 1 \mathrm{~b}}(t, s)\right)\right. \\
& +\frac{3}{2}\left(s^{2} A_{\mathrm{M}}^{1 \mathrm{a} \times 1 \mathrm{~b}}(s, t)+t^{2} A_{\mathrm{M}}^{1 \mathrm{a} \times 1 \mathrm{~b}}(t, s)\right) \\
& \left.\left.+2 s t\left(A_{\mathrm{M}}^{1 \mathrm{a} \times 1 \mathrm{~b}}(s, t)+A_{\mathrm{M}}^{1 \mathrm{a} \times 1 \mathrm{~b}}(t, s)\right)\right]\right\} .
\end{aligned}
$$

The auxiliary function $A^{1 \mathrm{a} \times 1 \mathrm{~b}}(x, y)$ is given by the product of $F_{\mathrm{V}}^{(1)}(x)$ and $F_{\mathrm{M}}^{(1)}(x)$, the renormalized one-loop vector (see Eq. (3.10)) and magnetic (vanishing in the high-energy limit) form factors for the QED vertex, and the complex-conjugate renormalized one-loop vacuumpolarization function $\Pi_{f}^{(1)}(x)$ (see Eq. (2.18)),

$$
A_{\mathrm{I}}^{1 \mathrm{a} \times 1 \mathrm{~b}}(x, y) \equiv \sum_{f} Q_{f}^{2} \operatorname{Re}\left\{F_{\mathrm{I}}^{(1)}(x)\left[\Pi_{f}^{(1)}(y)\right]^{\star}\right\}, \quad \mathrm{I}=\mathrm{V}, \mathrm{M} .
$$

Finally, the interference of diagrams of class 1a with those of class 1c gives

$$
\frac{d \sigma^{1 \mathrm{a} \times 1 \mathrm{c}}}{d \Omega}=\frac{\alpha^{2}}{4 s}\left[\frac{1}{s} A_{1}^{1 \mathrm{a} \times 1 \mathrm{c}}(s, t)+\frac{1}{t} A_{2}^{1 \mathrm{a} \times 1 \mathrm{c}}(s, t)\right] .
$$

Here the auxiliary functions $A_{1}^{1 \mathrm{a} \times 1 \mathrm{c}}(s, t)$ and $A_{2}^{1 \mathrm{a} \times 1 \mathrm{c}}(s, t)$ take the form

$$
\begin{aligned}
A_{1}^{1 \mathrm{a} \times 1 \mathrm{c}}(s, t)= & F_{\epsilon} \sum_{f} Q_{f}^{2} \operatorname{Re}\left\{\left[B_{\mathrm{A}}^{(1)}(s, t)+B_{\mathrm{B}}^{(1)}(t, s)+B_{\mathrm{C}}^{(1)}(u, t)\right.\right. \\
& \left.\left.-B_{\mathrm{B}}^{(1)}(u, s)\right]\left[\Pi_{f}^{(1)}(s)\right]^{\star}\right\}, \\
A_{2}^{1 \mathrm{a} \times 1 \mathrm{c}}(s, t)= & F_{\epsilon} \sum_{f} Q_{f}^{2} \operatorname{Re}\left\{\left[B_{\mathrm{B}}^{(1)}(s, t)+B_{\mathrm{A}}^{(1)}(t, s)-B_{\mathrm{B}}^{(1)}(u, t)\right.\right. \\
& \left.\left.+B_{\mathrm{C}}^{(1)}(u, s)\right]\left[\Pi_{f}^{(1)}(t)\right]^{\star}\right\}, \\
A_{1}^{1 \mathrm{a} \times 1 \mathrm{c}}(s, t)= & F_{\epsilon} \sum_{f} Q_{f}^{2} \operatorname{Re}\left\{\left[B_{\mathrm{A}}^{(1)}(s, t)+B_{\mathrm{B}}^{(1)}(t, s)+B_{\mathrm{C}}^{(1)}(u, t)\right.\right. \\
& \left.\left.-B_{\mathrm{B}}^{(1)}(u, s)\right]\left[\Pi_{f}^{(1)}(s)\right]^{\star}\right\},
\end{aligned}
$$




$$
\begin{aligned}
A_{2}^{1 \mathrm{a} \times 1 \mathrm{c}}(s, t)= & F_{\epsilon} \sum_{f} Q_{f}^{2} \operatorname{Re}\left\{\left[B_{\mathrm{B}}^{(1)}(s, t)+B_{\mathrm{A}}^{(1)}(t, s)-B_{\mathrm{B}}^{(1)}(u, t)\right.\right. \\
& \left.\left.+B_{\mathrm{C}}^{(1)}(u, s)\right]\left[\Pi_{f}^{(1)}(t)\right]^{\star}\right\} .
\end{aligned}
$$

$\Pi_{f}^{(1)}(x)$ is given in Eq. (2.18), and the new functions, in the small mass limit, read as

$$
\begin{aligned}
B_{\mathrm{A}}^{(1)}(x, y)= & -\frac{4}{\epsilon}\left(\frac{x^{2}}{y}+2 x+y\right) L_{e}(x)+\frac{x^{2}}{y}\left[16 \zeta_{2}+4 L_{e}(x)+2 L_{e}^{2}(y)-4 L_{e}(x) L_{e}(y)\right] \\
& +2 x\left[10 \zeta_{2}+L_{e}(x)+L_{e}(y)-L_{e}^{2}(x)+L_{e}^{2}(y)-2 L_{e}(x) L_{e}(y)\right] \\
& +y\left[10 \zeta_{2}+2 L_{e}(x)+2 L_{e}(y)-L_{e}^{2}(x)+L_{e}^{2}(y)-2 L_{e}(x) L_{e}(y)\right] \\
B_{\mathrm{B}}^{(1)}(x, y)= & -\frac{4}{\epsilon}\left(2 \frac{x^{2}}{y}+2 x+y\right) L_{e}(x)+4 \frac{x^{2}}{y}\left[8 \zeta_{2}+L_{e}^{2}(y)-2 L_{e}(x) L_{e}(y)\right] \\
& +2 x\left[10 \zeta_{2}-L_{e}(x)+L_{e}(y)-L_{e}^{2}(x)+L_{e}^{2}(y)-2 L_{e}(x) L_{e}(y)\right] \\
& +y\left[10 \zeta_{2}+2 L_{e}(x)+2 L_{e}(y)-L_{e}^{2}(x)+L_{e}^{2}(y)-2 L_{e}(x) L_{e}(y)\right] \\
& 4 x^{2} \\
B_{\mathrm{C}}^{(1)}(x, y)= & \frac{x^{2}}{y} L_{e}(x)+2 \frac{x^{2}}{y}\left[-8 \zeta_{2}-2 L_{e}(x)-L_{e}^{2}(y)+2 L_{e}(x) L_{e}(y)\right] \\
& -4(x+y) L_{e}(x) .
\end{aligned}
$$

For the computation of the non-fermionic corrections these functions are needed up to first order in $\epsilon$, since they are combined with the real emission. However, this higher-order expansion is not relevant here.

\section{The net fermionic NNLO differential cross section}

In this section we use the results of Section 3 and derive an explicit expression for the NNLO differential cross section of Eq. (2.19).

Note that the full set of two-loop fermionic virtual corrections to Bhabha scattering represents an infrared-divergent quantity. In order to obtain a finite quantity, we take into account the real emission of soft photons ${ }^{3}$ from the external legs of one-loop fermionic diagrams (class 1a, Fig. 1). The exact result is available in the literature, see, e.g., Eq. (25) and Appendix A in [18]. Here we show the high-energy approximation relevant for our computation. We consider events involving a single soft photon carrying energy $\omega$ in the final state,

$$
e^{-}\left(p_{1}\right)+e^{+}\left(p_{2}\right) \rightarrow e^{-}\left(p_{3}\right)+e^{+}\left(p_{4}\right)+\gamma(k),
$$

and compute one-loop purely-fermionic corrections. Obviously, these real corrections factorize and their structure is completely equivalent to the tree-level ones. In complete analogy with Eq. (2.6) we write

$$
\frac{d \sigma_{\gamma}}{d \Omega}=\left(\frac{\alpha}{\pi}\right) \frac{d \sigma_{\gamma}^{\mathrm{LO}}}{d \Omega}+\left(\frac{\alpha}{\pi}\right)^{2} \frac{d \sigma_{\gamma}^{\mathrm{NLO}}}{d \Omega}+\mathcal{O}\left(\alpha^{5}\right),
$$

\footnotetext{
3 The energy $\omega$ carried by a soft photon in the final state is small with respect to the center-of-mass energy $E$ introduced in Eq. (2.2).
} 
where

$$
\begin{aligned}
\frac{d \sigma_{\gamma}^{\mathrm{LO}}}{d \Omega}=\frac{\alpha^{2}}{s}\left[\frac{1}{2 s^{2}} v_{1}(s, t ; \epsilon)+\frac{1}{2 t^{2}} v_{1}(t, s ; \epsilon)+\frac{1}{s t} v_{2}(s, t ; \epsilon)\right] F\left(\omega, s, t, m_{e}^{2}\right), \\
\frac{d \sigma_{\gamma}^{\mathrm{NLO}}}{d \Omega}=\frac{\alpha^{2}}{s}\left\{\frac{1}{s^{2}} v_{1}(s, t ; \epsilon) \sum_{f} Q_{f}^{2} \operatorname{Re}\left[\Pi_{f}^{(1)}(s)\right]+\frac{1}{t^{2}} v_{1}(t, s ; \epsilon) \sum_{f} Q_{f}^{2} \operatorname{Re}\left[\Pi_{f}^{(1)}(t)\right]\right. \\
\left.+\frac{1}{s t} v_{2}(s, t ; \epsilon) \sum_{f} Q_{f}^{2} \operatorname{Re}\left[\Pi_{f}^{(1)}(s)+\Pi_{f}^{(1)}(t)\right]\right\} F\left(\omega, s, t, m_{e}^{2}\right) .
\end{aligned}
$$

$\Pi_{f}^{(1)}(x)$ can be read in Eq. (2.18) and, at variance with Eqs. (2.5)-(2.7), the kinematical factors introduced in Eq. (3.1) need to be expanded up to $\mathcal{O}(\epsilon)$, since the real-emission factor shows an infrared divergency,

$$
\begin{aligned}
F\left(\omega, s, t, m_{e}^{2}\right)= & F_{\epsilon}\left\{-\frac{2}{\epsilon}\left[\ln \left(\frac{s}{m_{e}^{2}}\right)+\ln \left(-\frac{t}{s}\right)-\ln \left(1+\frac{t}{s}\right)-1\right]\right. \\
& +\ln ^{2}\left(\frac{s}{m_{e}^{2}}\right)+2 \ln \left(\frac{s}{m_{e}^{2}}\right)\left[2 \ln \left(\frac{2 \omega}{\sqrt{s}}\right)+\ln \left(-\frac{t}{s}\right)-\ln \left(1+\frac{t}{s}\right)\right] \\
& +4 \ln \left(\frac{2 \omega}{\sqrt{s}}\right)\left[\ln \left(-\frac{t}{s}\right)-\ln \left(1+\frac{t}{s}\right)-1\right] \\
& -4 \zeta_{2}+\ln ^{2}\left(-\frac{t}{s}\right)-\ln ^{2}\left(1+\frac{t}{s}\right) \\
& \left.-2 \operatorname{Li}_{2}\left(-\frac{t}{s}\right)+2 \operatorname{Li}_{2}\left(1+\frac{t}{s}\right)\right\} .
\end{aligned}
$$

Summing the virtual contributions of Eq. (2.19) to the real-photon emission of Eq. (4.4) we write the NNLO fermionic corrections to Bhabha scattering through the sum of electron-loop contributions $\left(d \sigma^{\mathrm{NNLO}, \mathrm{e}}\right)$ and components arising from heavier fermion loops,

$$
\begin{aligned}
\frac{d \sigma^{\mathrm{NNLO}}}{d \Omega}+\frac{d \sigma_{\gamma}^{\mathrm{NLO}}}{d \Omega}= & \frac{d \sigma^{\mathrm{NNLO}, \mathrm{e}}}{d \Omega}+\sum_{f \neq e} Q_{f}^{2} \frac{d \sigma^{\mathrm{NNLO}, \mathrm{f}^{2}}}{d \Omega}+\sum_{f \neq e} Q_{f}^{4} \frac{d \sigma^{\mathrm{NNLO}, \mathrm{f}^{4}}}{d \Omega} \\
& +\sum_{f_{1}, f_{2} \neq e} Q_{f_{1}}^{2} Q_{f_{2}}^{2} \frac{d \sigma^{\mathrm{NNLO}, 2 \mathrm{f}}}{d \Omega} .
\end{aligned}
$$

The double summation over the fermion species arises from the loop-by-loop terms of Eqs. (3.6) and (3.24). Here we do not include the case $f_{1}=f_{2}=e$, which is incorporated in $d \sigma^{\mathrm{NNLO} \text {, e }}$. Note also the term proportional to $Q_{f}^{4}$, coming from Eq. (3.5). The result for electron loops can be found in Eq. (46) of [18]. For heavier fermion loops we introduce $x=-t / s$ and get:

$$
\begin{aligned}
\frac{d \sigma^{\mathrm{NNLO}, \mathrm{f}^{4}}}{d \Omega}= & \frac{\alpha^{2}}{2 s}\left\{\frac{\left(1-x+x^{2}\right)^{2}}{x^{2}}\left[\ln \left(\frac{s}{m_{e}^{2}}\right)+\ln \left(R_{f}\right)+4 \zeta_{3}-\frac{5}{6}\right]\right. \\
& \left.+\ln (x)\left(\frac{1}{x^{2}}-\frac{3}{2 x}+\frac{3}{2}-\frac{x}{2}\right)\right\},
\end{aligned}
$$




$$
\begin{aligned}
\frac{d \sigma^{\mathrm{NNLO}, 2 \mathrm{f}}}{d \Omega}= & \frac{\alpha^{2}}{s}\left\{\frac { ( 1 - x + x ^ { 2 } ) ^ { 2 } } { 3 x ^ { 2 } } \left[\ln ^{2}\left(\frac{s}{m_{e}^{2}}\right)+\ln \left(R_{f_{1}}\right) \ln \left(R_{f_{2}}\right)\right.\right. \\
& \left.+\ln \left(\frac{s}{m_{e}^{2}}\right)\left(\ln \left(R_{f_{1}}\right)+\ln \left(R_{f_{2}}\right)-\frac{10}{3}\right)-\frac{5}{3}\left(\ln \left(R_{f_{1}}\right)+\ln \left(R_{f_{2}}\right)-\frac{5}{3}\right)\right] \\
& +\frac{1}{3} \ln ^{2}(x)\left(\frac{1}{x^{2}}-\frac{4}{3 x}+\frac{7}{6}-\frac{x}{3}\right)+\frac{\zeta_{2}}{3}\left(\frac{2}{x}-5+4 x-2 x^{2}\right) \\
& \left.+\ln (x)\left[\ln \left(R_{f_{1}}\right)+\ln \left(R_{f_{2}}\right)-\frac{10}{3}+2 \ln \left(\frac{s}{m_{e}^{2}}\right)\right]\left(\frac{1}{3 x^{2}}-\frac{1}{2 x}+\frac{1}{2}-\frac{x}{6}\right)\right\}
\end{aligned}
$$

$$
\begin{aligned}
& \frac{d \sigma^{\mathrm{NNLO}, \mathrm{f}^{2}}}{d \Omega}=\frac{\alpha^{2}}{s}\left\{\sigma_{1}^{\mathrm{NNLO}, \mathrm{f}^{2}}+\sigma_{2}^{\mathrm{NNLO}, \mathrm{f}^{2}} \ln \left(\frac{2 \omega}{\sqrt{s}}\right)\right\}, \\
& \sigma_{1}^{\mathrm{NNLO}, \mathrm{f}^{2}}=\frac{\left(1-x+x^{2}\right)^{2}}{3 x^{2}}\left\{-\frac{1}{3}\left[\ln ^{3}\left(\frac{s}{m_{e}^{2}}\right)+\ln ^{3}\left(R_{f}\right)\right]+\ln ^{2}\left(\frac{s}{m_{e}^{2}}\right)\left[\frac{55}{6}-\ln \left(R_{f}\right)\right.\right. \\
& +\ln (1-x)-\ln (x)]+\ln \left(\frac{s}{m_{e}^{2}}\right)\left[-\frac{589}{18}+\frac{37}{3} \ln \left(R_{f}\right)-\ln ^{2}\left(R_{f}\right)\right. \\
& \left.-2 \ln \left(R_{f}\right)(\ln (x)-\ln (1-x))-8 \operatorname{Li}_{2}(x)\right] \\
& +\frac{4795}{108}-\frac{409}{18} \ln \left(R_{f}\right)+\frac{19}{6} \ln ^{2}\left(R_{f}\right) \\
& \left.-\ln ^{2}\left(R_{f}\right)(\ln (x)-\ln (1-x))-8 \ln \left(R_{f}\right) \mathrm{Li}_{2}(x)+\frac{40}{3} \mathrm{Li}_{2}(x)\right\} \\
& +\ln \left(\frac{s}{m_{e}^{2}}\right)\left[\zeta_{2}\left(-\frac{2}{3 x^{2}}+\frac{4}{3 x}+\frac{11}{2}-\frac{23}{3} x+\frac{16}{3} x^{2}\right)+\ln ^{2}(x)\left(-\frac{1}{3 x^{2}}\right.\right. \\
& \left.+\frac{17}{12 x}-\frac{5}{4}-\frac{x}{12}+\frac{2}{3} x^{2}\right)+\ln ^{2}(1-x)\left(-\frac{2}{3 x^{2}}+\frac{11}{6 x}-\frac{5}{2}+\frac{11}{6} x-\frac{2}{3} x^{2}\right) \\
& +\ln (x) \ln (1-x)\left(\frac{2}{3 x^{2}}-\frac{4}{3 x}-\frac{1}{2}+\frac{5}{3} x-\frac{4}{3} x^{2}\right)+\ln (x)\left(\frac{55}{9 x^{2}}-\frac{83}{9 x}+\frac{65}{6}\right. \\
& \left.\left.-\frac{85}{18} x+\frac{10}{9} x^{2}\right)+\frac{1}{3} \ln (1-x)\left(-\frac{10}{3 x^{2}}+\frac{31}{6 x}-10+\frac{31}{6} x-\frac{10}{3} x^{2}\right)\right] \\
& +\frac{1}{3} \ln ^{3}(x)\left(-\frac{1}{3 x^{2}}+\frac{31}{12 x}-\frac{11}{6}-\frac{x}{6}+\frac{x^{2}}{3}\right)+\frac{1}{3} \ln ^{3}(1-x)\left(-\frac{1}{3 x^{2}}+\frac{1}{x}\right. \\
& \left.-\frac{4}{3}+x-\frac{x^{2}}{3}\right)+\ln ^{2}(x) \ln (1-x)\left(-\frac{1}{3 x^{2}}+\frac{1}{3 x}-\frac{4}{3}+x-\frac{x^{2}}{3}\right) \\
& +\frac{1}{3} \ln (x) \ln ^{2}(1-x)\left(-\frac{1}{x^{2}}+\frac{2}{x}-\frac{7}{4}+\frac{x}{2}\right)+\ln ^{2}(x)\left[\frac{55}{18 x^{2}}-\frac{46}{9 x}\right. \\
& \left.+\frac{14}{3}-\frac{4}{9} x-\frac{10}{9} x^{2}+\ln \left(R_{f}\right)\left(-\frac{1}{3 x^{2}}+\frac{17}{12 x}-\frac{5}{4}-\frac{x}{12}+\frac{2}{3} x^{2}\right)\right] \\
& +\ln ^{2}(1-x)\left[\frac{10}{9 x^{2}}-\frac{29}{9 x}+\frac{9}{2}-\frac{29}{9} x+\frac{10}{9} x^{2}+\ln \left(R_{f}\right)\left(-\frac{2}{3 x^{2}}+\frac{11}{6 x}\right.\right.
\end{aligned}
$$




$$
\begin{aligned}
& \left.\left.-\frac{5}{2}+\frac{11}{6} x-\frac{2}{3} x^{2}\right)\right]+\ln (x) \ln (1-x)\left[-\frac{10}{9 x^{2}}+\frac{37}{18 x}+\frac{1}{2}-\frac{25}{9} x\right. \\
& \left.+\frac{20}{9} x^{2}+\ln \left(R_{f}\right)\left(\frac{2}{3 x^{2}}-\frac{4}{3 x}-\frac{1}{2}+\frac{5}{3} x-\frac{4}{3} x^{2}\right)\right] \\
& +\ln (x)\left[-\frac{589}{54 x^{2}}+\frac{1753}{108 x}-\frac{701}{36}+\frac{925}{108} x-\frac{56}{27} x^{2}\right. \\
& +\operatorname{Li}_{2}(x)\left(-\frac{4}{x^{2}}+\frac{19}{3 x}-7+3 x-\frac{2}{3} x^{2}\right) \\
& +\ln \left(R_{f}\right)\left(\frac{37}{9 x^{2}}-\frac{56}{9 x}+\frac{47}{6}-\frac{67}{18} x+\frac{10}{9} x^{2}\right)+\zeta_{2}\left(-\frac{2}{3 x^{2}}+\frac{4}{x}-\frac{1}{6}\right. \\
& \left.\left.-\frac{10}{3} x+2 x^{2}\right)\right]+\ln (1-x)\left[\frac{56}{27 x^{2}}-\frac{161}{54 x}+\frac{56}{9}-\frac{161}{54} x+\frac{56}{27} x^{2}\right. \\
& +\ln \left(R_{f}\right)\left(-\frac{10}{9 x^{2}}+\frac{31}{18 x}-\frac{10}{3}+\frac{31}{18} x-\frac{10}{9} x^{2}\right)+\zeta_{2}\left(-\frac{2}{x^{2}}+\frac{20}{3 x}\right. \\
& \left.\left.-\frac{32}{3}+\frac{20}{3} x-2 x^{2}\right)\right]+\operatorname{Li}_{3}(x)\left(\frac{4}{3 x^{2}}-\frac{7}{3 x}+3-\frac{5}{3} x+\frac{2}{3} x^{2}\right) \\
& +\frac{2}{3} S_{1,2}(x)\left(-\frac{1}{x^{2}}+\frac{1}{x}-x+x^{2}\right)+\zeta_{2}\left[\frac{19}{9 x^{2}}-\frac{13}{18 x}-\frac{43}{3}\right. \\
& \left.+\frac{311}{18} x-\frac{98}{9} x^{2}+\ln \left(R_{f}\right)\left(-\frac{2}{3 x^{2}}+\frac{4}{3 x}+\frac{11}{2}-\frac{23}{3} x+\frac{16}{3} x^{2}\right)\right] \\
& +\zeta_{3}\left(-\frac{4}{3 x^{2}}+\frac{3}{x}-5+\frac{11}{3} x-2 x^{2}\right) \text {, } \\
& \sigma_{2}^{\mathrm{NNLO}, \mathrm{f}^{2}}=\frac{8}{3} \frac{\left(1-x+x^{2}\right)^{2}}{x^{2}}\left\{\ln ^{2}\left(\frac{s}{m_{e}^{2}}\right)+\ln \left(\frac{s}{m_{e}^{2}}\right)\left[-\frac{8}{3}+\ln \left(R_{f}\right)\right.\right. \\
& \left.-\ln (1-x)]+\ln (x) \ln \left(R_{f}\right)+\left[\frac{5}{3}-\ln \left(R_{f}\right)\right][1+\ln (1-x)]\right\} \\
& +4\left[\ln \left(\frac{s}{m_{e}^{2}}\right) \ln (x)\left(\frac{4}{3 x^{2}}-\frac{7}{3 x}+3-\frac{5}{3} x+\frac{2}{3} x^{2}\right)+\ln ^{2}(x)\left(\frac{2}{3 x^{2}}-\frac{1}{x}\right.\right. \\
& \left.+1-\frac{1}{3} x\right)-\ln (x) \ln (1-x)\left(\frac{2}{3 x^{2}}-\frac{1}{x}+1-\frac{1}{3} x\right) \\
& \left.-\frac{1}{3} \ln (x)\left(\frac{16}{3 x^{2}}-\frac{29}{3 x}+13-\frac{23}{3} x+\frac{10}{3} x^{2}\right)\right] \text {. }
\end{aligned}
$$

In order to have compact results we used

$$
S_{n, p}(y)=\frac{(-1)^{n+p-1}}{(n-1) ! p !} \int_{0}^{1} d x \frac{\ln ^{n-1}(x) \ln ^{p}(1-x y)}{x} .
$$

In Table 4 (Table 5) we show numerical values for the NNLO corrections to the differential cross section for a scattering angle $\theta=3^{\circ}\left(\theta=90^{\circ}\right)$. In both tables we set $\omega=E / 10$. Finally, in 
Table 4

Numerical values for the NNLO corrections to the differential cross section respect to the solid angle. Results are expressed in nanobarns for a scattering angle $\theta=3^{\circ}$. Empty entries are related to cases where the high-energy approximation cannot be applied

\begin{tabular}{llcc}
\hline$d \sigma / d \Omega[\mathrm{nb}] \mid \sqrt{s}[\mathrm{GeV}]$ & 10 & 91 & 500 \\
\hline LO QED [Eq. $(2.5)]$ & 440873 & 5323.91 & 176.349 \\
LO Zfitter [51,52] & 440875 & 5331.5 & 176.283 \\
NNLO $(e)[$ Eq. $(4.6)]$ & -1397.35 & -35.8374 & -1.88151 \\
NNLO $(e+\mu)[$ Eq. $(4.6)]$ & -1394.74 & -43.1888 & -2.41643 \\
NNLO $(e+\mu+\tau)[$ Eq. $(4.6)]$ & & & -2.55179 \\
NNLO photonic $[14,16]$ & 9564.09 & 251.661 & 12.7943 \\
\hline
\end{tabular}

Table 5

Numerical values for the NNLO corrections to the differential cross section respect to the solid angle. Results are expressed in nanobarns for a scattering angle $\theta=90^{\circ}$. Empty entries are related to cases where the high-energy approximation cannot be applied

\begin{tabular}{lccc}
\hline$d \sigma / d \Omega$ [nb] $\mid \sqrt{s}[\mathrm{GeV}]$ & 10 & 91 & 500 \\
\hline LO QED [Eq. (2.5)] & 0.466409 & 0.00563228 & 0.000186564 \\
LO Zfitter [51,52] & 0.468499 & 0.127292 & 0.0000854731 \\
NNLO $(e)$ [Eq. (4.6)] & -0.00453987 & -0.0000919387 & $-4.28105 \times 10^{-6}$ \\
NNLO $(e+\mu)$ [Eq. (4.6)] & -0.00570942 & -0.000122796 & $-5.90469 \times 10^{-6}$ \\
NNLO $(e+\mu+\tau)[$ Eq. (4.6)] & -0.00586082 & -0.000135449 & $-6.7059 \times 10^{-6}$ \\
NNLO $(e+\mu+\tau+t)[$ Eq. (4.6)] & & & $-6.6927 \times 10^{-6}$ \\
NNLO photonic [14,16] & 0.0358755 & 0.000655126 & 0.0000284063 \\
\hline
\end{tabular}
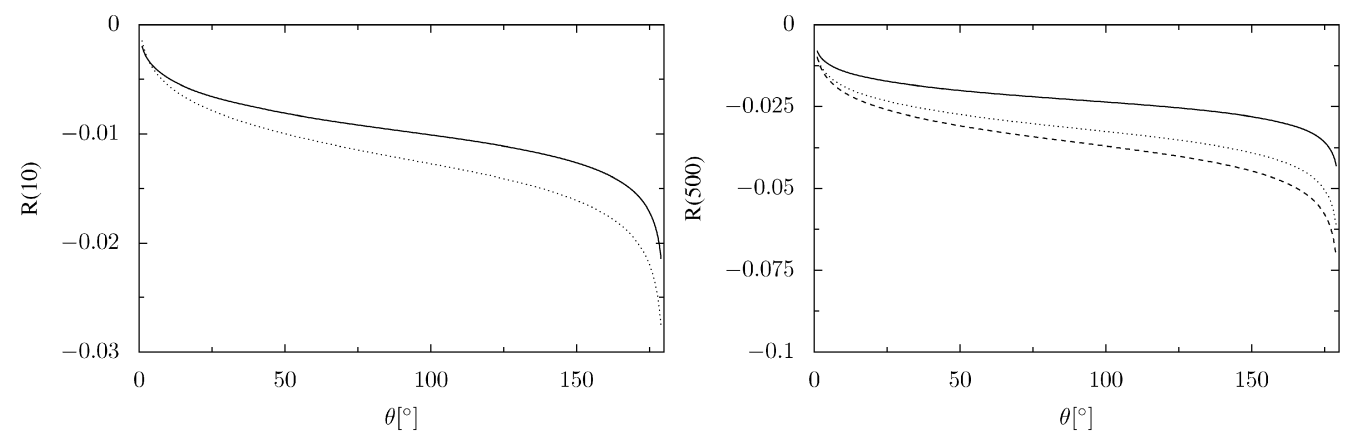

Fig. 4. Ratio of the fermionic NNLO corrections to the differential cross section respect to the tree-level result for $\sqrt{s}=10 \mathrm{GeV}$ and $\sqrt{s}=500 \mathrm{GeV}$. A solid line represents the electron-loop contributions, a dotted one the sum of electron- and muon-loop ones, and a dashed one includes also $\tau$ leptons.

Fig. 4 we plot the ratio of the two-loop fermionic corrections to the tree-level cross section,

$$
R(\sqrt{s})=\left(\frac{\alpha}{\pi}\right)^{2} \frac{d \sigma^{\mathrm{NNLO}}+d \sigma_{\gamma}^{\mathrm{NLO}}}{d \sigma^{\mathrm{LO}}}
$$

for $\sqrt{s}=10 \mathrm{GeV}$ and $\sqrt{s}=500 \mathrm{GeV}$ (see also Fig. 5).

It is clear from the tables, that although there is no decoupling of the heavier fermions (as indeed there should not, since the typical scale of the process is large compared to all the masses), 

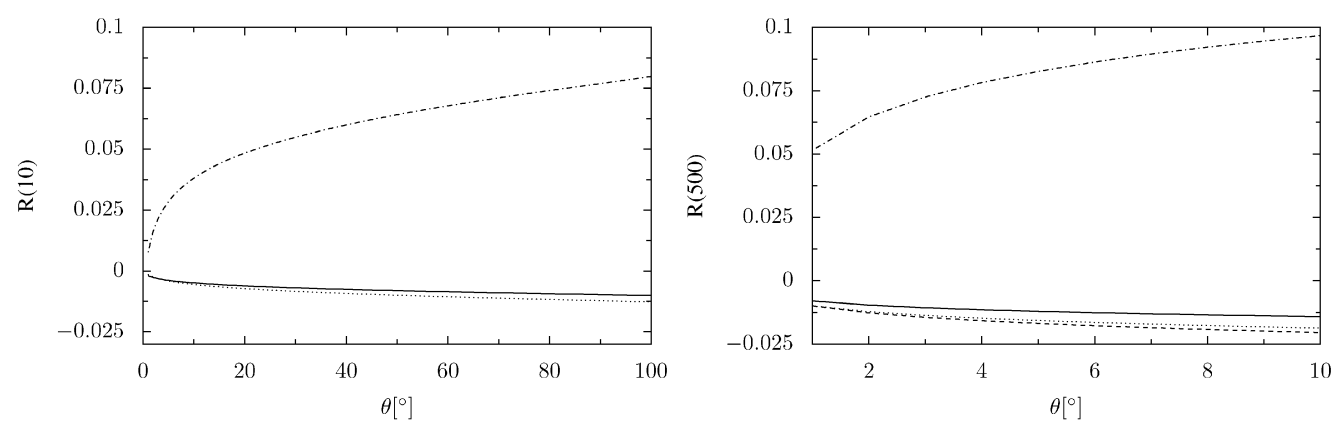

Fig. 5. Same as Fig. 4, including the photonic contributions of $[2,14,16]$ (dash-dotted lines).

the electron loop contributions dominate in the fermionic part and the latter is still substantially smaller than the pure photonic corrections.

\section{Summary}

In this article, we completed the computation of the virtual two-loop QED fermionic corrections to Bhabha scattering. Based on the kinematics of the targeted phenomenological applications, we considered the limit $m_{e}^{2} \ll m_{f}^{2} \ll s, t, u$.

The fermionic double box contributions with two different mass scales have been derived for the first time here. Their numerical importance is comparable to the two-loop self-energies and vertices. We note, however, a qualitative difference. Due to the structure of the collinear singularities of the graphs, the contributions of the heavier fermions are not suppressed.

A numerical estimation of differential cross sections shows that the net fermionic two-loop effects may be neglected for applications at LEP 1 and LEP 2, but have to be taken into account for precision calculations when a level of $10^{-4}$ has to be reached, as is anticipated for the Giga-Z option of the ILC project.

Completing the NNLO program for Bhabha scattering requires still several ingredients. First, let us mention the contributions from the five light quark flavors. Here, an approach based on dispersion relations à $l a$ [53] should be suitable. On the other hand, the heavy top quark might be considered decoupling in a large part of the interesting kinematical regions. Furthermore, an implementation of the loop-by-loop corrections with pentagon diagrams has to be done.

Finally, light fermionic pair emission diagrams need to be considered. As known from the form-factor case, they are responsible for the cancellation of the leading part of the logarithmic sensitivity on the masses.

Exact and approximated results are made publicly available at [26]. The combination of our result with the photonic two-loop corrections of [16] and with electron loop corrections of [17, 25] proves well-suited for phenomenological purposes, e.g., a precise luminosity determination at a future International Linear Collider.

\section{Note added}

We would like to thank T. Becher and K. Melnikov for drawing our attention to a problem with a first version of our result, which lead us to discover incorrectly expanded integrals (Eqs. (A.7), (A.8) and (A.15) in Appendix A) appearing in the evaluation of the two-loop box form factors 
(Eqs. (3.20), (3.21) and (3.22) of Section 3.3). After correction, Eq. (4.10) agrees with the result published in the meantime in [55].

\section{Acknowledgements}

We would like to thank A. Arbuzov, R. Bonciani, A. Ferroglia and A. Penin for useful communications, and S. Moch and A. Mitov for interesting discussions.

Work supported in part by Sonderforschungsbereich/Transregio TRR 9 of DFG 'Computergestützte Theoretische Teilchenphysik', by the Sofja Kovalevskaja Award of the Alexander von Humboldt Foundation sponsored by the German Federal Ministry of Education and Research, by the ToK Program "ALGOTOOLS" (MTKD-CD-2004-014319), by the Polish State Committee for Scientific Research (KBN, research projects in 2004-2006), and by the European Community's Marie-Curie Research Training Networks MRTN-CT-2006-035505 'HEPTOOLS' and MRTN-CT-2006-035482 'FLAVIAnet'.

\section{Appendix A. Mass-expanded master integrals}

The list of master integrals required by our computation can already be found in Table $\mathrm{V}$ of [22]. The eight most difficult masters, those involving two different mass scales, have been derived in [44]. Because they are a substantial part of the present study we reproduce them here:

SE312M1m[on shel1]

$$
\begin{aligned}
= & M^{2} m^{-4 \epsilon}\left\{R\left[\frac{1}{2 \epsilon^{2}}+\frac{5}{4 \epsilon}-\frac{3}{8}+\frac{\zeta_{2}}{2}+\frac{3}{2} L(R)-\frac{1}{2} L^{2}(R)\right]\right. \\
& +R^{2}\left[\frac{11}{18}-\frac{1}{3} L(R)\right]+\epsilon\left[R \left(\frac{45}{16}+\frac{5}{4} \zeta_{2}-\frac{\zeta_{3}}{3}-\frac{7}{4} L(R)+L^{2}(R)\right.\right. \\
& \left.\left.\left.-\frac{1}{2} L^{3}(R)\right)+R^{2}\left(-\frac{3}{4}+\frac{8}{9} L(R)-\frac{1}{2} L^{2}(R)\right)\right]\right\},
\end{aligned}
$$

SE3 12M1ma[on shel1]

$$
\begin{aligned}
= & m^{-4 \epsilon}\left\{\frac{1}{2 \epsilon^{2}}+\frac{1}{2 \epsilon}[1+2 L(R)]+\frac{1}{2}\left(1+\zeta_{2}\right)+L(R)+L^{2}(R)\right. \\
& +\epsilon\left[\frac{1}{6}\left(3+3 \zeta_{2}-2 \zeta_{3}\right)+\left(1+\zeta_{2}\right) L(R)+L^{2}(R)+\frac{2}{3} L^{3}(R)\right] \\
& +R\left[-\frac{3}{4}+\frac{1}{2} L(R)+\epsilon\left(\frac{7}{8}-L(R)+\frac{3}{4} L^{2}(R)\right)\right] \\
& \left.+R^{2}\left[-\frac{5}{36}+\frac{1}{6} L(R)+\epsilon\left(-\frac{5}{72}+\frac{1}{18} L(R)+\frac{1}{4} L^{2}(R)\right)\right]\right\} .
\end{aligned}
$$

In the following we set $L_{m}(x)=\ln \left(-m^{2} / x\right)$ and $L_{M}(x)=\ln \left(-M^{2} / x\right)$,

$$
\begin{aligned}
\mathrm{V} 412 \mathrm{M} 1 \mathrm{~m}[\mathrm{x}]= & m^{-4 \epsilon}\left\{\frac{1}{2 \epsilon^{2}}+\frac{5}{2 \epsilon}+\frac{1}{2}\left[19-3 \zeta_{2}-L_{m}^{2}(x)\right]\right. \\
& +\frac{M^{2}}{x}\left[-2+4 \zeta_{2}-4 \zeta_{3}-2 L_{m}(x)+2 L_{M}(x)-4 \zeta_{2} L_{M}(x)\right. \\
& \left.\left.+2 L_{m}(x) L_{M}(x)-L_{M}^{2}(x)-L_{m}(x) L_{M}^{2}(x)+\frac{1}{3} L_{M}^{3}(x)\right]\right\},
\end{aligned}
$$




$$
\begin{aligned}
\mathrm{V} 412 \mathrm{M} 1 \mathrm{ma}[\mathrm{x}]= & \frac{m^{-4 \epsilon}}{m^{2}}\left\{\frac{1}{2 \epsilon^{2}}+\frac{1}{\epsilon}\left[1+\frac{1}{2} L_{m}(x)\right]+2-\zeta_{2}+L_{m}(x)+\frac{1}{4} L_{m}^{2}(x)\right. \\
& +\frac{M^{2}}{x}\left[\frac{1}{\epsilon}-\frac{1}{\epsilon} L_{M}(x)-1+3 \zeta_{2}+L_{m}(x)+L_{M}(x)\right. \\
& \left.\left.-L_{m}(x) L_{M}(x)-\frac{1}{2} L_{M}^{2}(x)\right]\right\}, \\
\mathrm{V} 412 \mathrm{M} 2 \mathrm{~m}[\mathrm{x}]=m^{-} & \left\{\frac{1}{2 \epsilon^{2}}+\frac{1}{\epsilon}\left[\frac{5}{2}+L_{m}(x)\right]+\frac{1}{2}\left(19+\zeta_{2}\right)+5 L_{m}(x)+L_{m}^{2}(x)\right\}, \\
\mathrm{V} 412 \mathrm{M} 2 \mathrm{ma}[\mathrm{x}]= & \frac{m^{-4 \epsilon}}{6 x}\left[12 \zeta_{3}-6 \zeta_{2} L_{M}(x)-L_{M}^{3}(x)\right], \\
\mathrm{B} 512 \mathrm{M} 2 \mathrm{~m}[\mathrm{x}, \mathrm{y}]= & \frac{m^{-4 \epsilon}}{x}\left\{\frac{1}{\epsilon^{2}} L_{m}(x)+\frac{1}{\epsilon}\left(-\zeta_{2}+2 L_{m}(x)+\frac{1}{2} L_{m}^{2}(x)+L_{m}(x) L_{m}(y)\right)\right. \\
& -2 \zeta_{2}-2 \zeta_{3}+4 L_{m}(x)+L_{m}^{2}(x)+\frac{1}{3} L_{m}^{3}(x)-4 \zeta_{2} L_{m}(y) \\
& +2 L_{m}(x) L_{m}(y)+L_{m}(x) L_{m}^{2}(y)-\frac{1}{6} L_{m}^{3}(y) \\
& -\left(3 \zeta_{2}+\frac{1}{2} L_{m}^{2}(x)-L_{m}(x) L_{m}(y)+\frac{1}{2} L_{m}^{2}(y)\right) \ln \left(1+\frac{y}{x}\right) \\
& \left.-\left(L_{m}(x)-L_{m}(y)\right) \mathrm{Li}_{2}\left(-\frac{y}{x}\right)+\mathrm{Li}_{3}\left(-\frac{y}{x}\right)\right\}, \\
& (\mathrm{A})\} \\
& \\
&
\end{aligned}
$$

$$
\begin{aligned}
\mathrm{B} 512 \mathrm{M} 2 \mathrm{ma}[\mathrm{x}, \mathrm{y}] \\
=\frac{m^{-4 \epsilon}}{x y}\left\{\frac{1}{\epsilon}\left[-L_{m}(x) L_{m}(y)+L_{m}(x) L(R)\right]-2 \zeta_{3}+\zeta_{2} L_{m}(x)+4 \zeta_{2} L_{m}(y)\right. \\
\quad-2 L_{m}(x) L_{m}^{2}(y)+\frac{1}{6} L_{m}^{3}(y)-2 \zeta_{2} L(R)+2 L_{m}(x) L_{m}(y) L(R)-\frac{1}{6} L^{3}(R) \\
\quad+\left(3 \zeta_{2}+\frac{1}{2} L_{m}^{2}(x)-L_{m}(x) L_{m}(y)+\frac{1}{2} L_{m}^{2}(y)\right) \ln \left(1+\frac{y}{x}\right) \\
\left.\quad+\left(L_{m}(x)-L_{m}(y)\right) \operatorname{Li}_{2}\left(-\frac{y}{x}\right)-\operatorname{Li}_{3}\left(-\frac{y}{x}\right)\right\} .
\end{aligned}
$$

We list also the other expanded masters, including the correct normalizations. Note that, compared to the conventions employed in [22] and in Eq. (2.16), all integrals are rescaled by a factor $m^{L(D-2 l)}$, where $L$ is the number of loops, $D=4-2 \epsilon$ and $l$ is the number of internal lines. Expansions are performed up to the order required by our computation. For example, we include $\mathcal{O}\left(m^{2}\right)$ terms in $\mathrm{SE} 212 \mathrm{~m}[\mathrm{x}]$ (see Eq. (A.10)) since the reduction procedure generates coefficients containing $1 / \mathrm{m}^{2}$. The same consideration applies to $\mathcal{O}(\epsilon)$ terms, which are included as long as the reduction brings inverse powers of $\epsilon$ in the coefficient functions. Since in the following no ambiguities arise, in we drop the subscript $f$ and we set $L(x)=\ln \left(-m^{2} / x\right)$,

$$
\mathrm{T} 111 \mathrm{~m}=\left(m^{2}\right)^{1-\epsilon}\left[\frac{1}{\epsilon}+1+\epsilon\left(1+\frac{\zeta_{2}}{2}\right)+\epsilon^{2}\left(1+\frac{\zeta_{2}}{2}-\frac{\zeta_{3}}{3}\right)\right],
$$


SE212m[x]

$$
\begin{aligned}
= & m^{-2 \epsilon}\left\{\frac{1}{\epsilon}+2+L(x)+2 \frac{m^{2}}{x}[1-L(x)]+\epsilon\left[4-\frac{\zeta_{2}}{2}+2 L(x)+\frac{1}{2} L^{2}(x)\right.\right. \\
& \left.+\frac{m^{2}}{x}\left(2+2 \zeta_{2}-L^{2}(x)\right)\right]+\epsilon^{2}\left[8-\zeta_{2}-\frac{7}{3} \zeta_{3}+4 L(x)-\frac{1}{2} \zeta_{2} L(x)+L^{2}(x)\right. \\
& \left.\left.+\frac{1}{6} L^{3}(x)+\frac{m^{2}}{x}\left(2+\zeta_{2}+4 \zeta_{3}+\zeta_{2} L(x)-\frac{1}{3} L^{3}(x)\right)\right]\right\},
\end{aligned}
$$

SE2 $10 \mathrm{~m}[\mathrm{x}]$

$$
\begin{aligned}
&= m^{-2 \epsilon}\left\{\frac{1}{\epsilon}+2+L(x)+\epsilon\left[4-\frac{\zeta_{2}}{2}+2 L(x)+\frac{1}{2} L^{2}(x)\right]\right. \\
&\left.+\epsilon^{2}\left[8-\zeta_{2}-\frac{7}{3} \zeta_{3}+4 L(x)-\frac{1}{2} \zeta_{2} L(x)+L^{2}(x)+\frac{1}{6} L^{3}(x)\right]\right\}, \\
& \text { V311m[x] }=\frac{m^{-2 \epsilon}}{x}\left\{4 \zeta_{2}+\frac{1}{2} L^{2}(x)-\epsilon\left[-5 \zeta_{3}+\zeta_{2} L(x)-\frac{1}{6} L^{3}(x)\right]\right\},
\end{aligned}
$$

SE311m[on shel1]

$$
\begin{aligned}
= & \left(m^{2}\right)^{1-2 \epsilon}\left[\frac{1}{2 \epsilon^{2}}+\frac{5}{4 \epsilon}+\frac{11}{8}+\frac{5}{2} \zeta_{2}+\epsilon\left(-\frac{55}{16}+\frac{25}{4} \zeta_{2}+\frac{11}{3} \zeta_{3}\right)\right. \\
& \left.+\epsilon^{2}\left(-\frac{949}{32}+\frac{55}{8} \zeta_{2}+\frac{55}{6} \zeta_{3}+\frac{303}{8} \zeta_{4}\right)\right],
\end{aligned}
$$

SE3 12m[x]

$$
\begin{aligned}
= & \left(m^{2}\right)^{1-2 \epsilon}\left\{\frac{1}{\epsilon^{2}}+\frac{1}{\epsilon}\left(3-\frac{x}{4 m^{2}}\right)+5+\zeta_{2}-L^{2}(x)-\frac{x}{m^{2}}\left[\frac{13}{8}+\frac{1}{2} L(x)\right]\right. \\
& +\epsilon\left[3+3 \zeta_{2}+\frac{16}{3} \zeta_{3}-4 L(x)+2 \zeta_{2} L(x)-3 L^{2}(x)-L^{3}(x)\right. \\
& \left.\left.-\frac{x}{m^{2}}\left(\frac{115}{16}-\frac{\zeta_{2}}{4}+\frac{13}{4} L(x)+\frac{1}{2} L^{2}(x)\right)\right]\right\},
\end{aligned}
$$

SE3 $12 \mathrm{md}[\mathrm{x}]$

$$
\begin{aligned}
= & m^{-4 \epsilon}\left\{\frac{1}{2 \epsilon^{2}}+\frac{1}{2 \epsilon}-\frac{1}{2}+\frac{\zeta_{2}}{2}-L(x)-\frac{1}{2} L^{2}(x)+\frac{m^{2}}{x}\left[-2+L^{2}(x)\right]\right. \\
& +\epsilon\left[-\frac{11}{2}+\frac{3}{2} \zeta_{2}+\frac{8}{3} \zeta_{3}-5 L(x)+\zeta_{2} L(x)-2 L^{2}(x)-\frac{1}{2} L^{3}(x)\right. \\
& \left.\left.+\frac{m^{2}}{x}\left(-6 \zeta_{3}-4 L(x)-2 \zeta_{2} L(x)+L^{2}(x)+L^{3}(x)\right)\right]\right\} .
\end{aligned}
$$

Finally, the mass expanded one-loop box master integral B $412 \mathrm{~m}[\mathrm{x}, \mathrm{y}]$ can be collected from Eqs. (4.70)-(4.75) of [54]: 


$$
\begin{array}{rl}
\mathrm{B} 4 & 12 \mathrm{~m}[\mathrm{x}, \mathrm{y}] \\
= & \frac{m^{-2 \epsilon}}{x y}\left\{\frac{2}{\epsilon}\left[L(y)-\ln \left(\frac{x}{y}\right)\right]+2 L^{2}(y)-2 L(y) \ln \left(\frac{x}{y}\right)+\epsilon\left[4 \zeta_{3}-9 \zeta_{2} L(y)\right.\right. \\
& +\frac{2}{3} L^{3}(y)+5 \zeta_{2} \ln \left(\frac{x}{y}\right)-L^{2}(y) \ln \left(\frac{x}{y}\right)+\frac{1}{3} \ln ^{3}\left(\frac{x}{y}\right)-6 \zeta_{2} \ln \left(1+\frac{x}{y}\right) \\
& +2 \ln \left(-\frac{x}{y}\right) \ln \left(\frac{x}{y}\right) \ln \left(1+\frac{x}{y}\right)-\ln ^{2}\left(\frac{x}{y}\right) \ln \left(1+\frac{x}{y}\right) \\
& \left.\left.+2 \ln \left(\frac{x}{y}\right) \operatorname{Li}_{2}\left(1+\frac{x}{y}\right)+2 \operatorname{Li}_{3}\left(-\frac{x}{y}\right)\right]\right\} .
\end{array}
$$

\section{References}

[1] D. Bardin, W. Hollik, T. Riemann, Z. Phys. C 49 (1991) 485-490.

[2] A. Arbuzov, E. Kuraev, B. Shaikhatdenov, Mod. Phys. Lett. A 13 (1998) 2305-2316, hep-ph/9806215.

[3] Z. Bern, L. Dixon, A. Ghinculov, Phys. Rev. D 63 (2001) 053007, hep-ph/0010075.

[4] V. Smirnov, Phys. Lett. B 460 (1999) 397-404, hep-ph/9905323.

[5] J. Tausk, Phys. Lett. B 469 (1999) 225-234, hep-ph/9909506.

[6] F. Berends, R. Kleiss, Nucl. Phys. B 228 (1983) 537.

[7] F. Berends, R. Kleiss, W. Hollik, Nucl. Phys. B 304 (1988) 712.

[8] S. Jadach, W. Placzek, E. Richter-Was, B. Ward, Z. Was, Comput. Phys. Commun. 102 (1997) 229-251.

[9] S. Jadach, W. Placzek, B. Ward, Phys. Lett. B 390 (1997) 298-308, hep-ph/9608412.

[10] A. Arbuzov, G. Fedotovich, E. Kuraev, N. Merenkov, V. Rushai, L. Trentadue, JHEP 9710 (1997) 001, hep$\mathrm{ph} / 9702262$.

[11] A. Arbuzov, G. Fedotovich, F. Ignatov, E. Kuraev, A. Sibidanov, Eur. Phys. J. C 46 (2006) 689-703, hep$\mathrm{ph} / 0504233$.

[12] C. Carloni Calame, C. Lunardini, G. Montagna, O. Nicrosini, F. Piccinini, Nucl. Phys. B 584 (2000) 459-479, hep-ph/0003268.

[13] G. Balossini, C. Carloni Calame, G. Montagna, O. Nicrosini, F. Piccinini, Nucl. Phys. B 758 (2006) 227-253, hep-ph/0607181.

[14] N. Glover, B. Tausk, J. van der Bij, Phys. Lett. B 516 (2001) 33-38, hep-ph/0106052.

[15] A. Penin, Phys. Rev. Lett. 95 (2005) 010408, hep-ph/0501120.

[16] A. Penin, Nucl. Phys. B 734 (2006) 185-202, hep-ph/0508127.

[17] R. Bonciani, A. Ferroglia, P. Mastrolia, E. Remiddi, J. van der Bij, Nucl. Phys. B 701 (2004) 121-179, hep$\mathrm{ph} / 0405275$.

[18] R. Bonciani, A. Ferroglia, P. Mastrolia, E. Remiddi, J. van der Bij, Nucl. Phys. B 716 (2005) 280-302, hep$\mathrm{ph} / 0411321$.

[19] R. Bonciani, A. Ferroglia, Phys. Rev. D 72 (2005) 056004, hep-ph/0507047.

[20] V.A. Smirnov, Phys. Lett. B 524 (2002) 129, hep-ph/0111160.

[21] G. Heinrich, V.A. Smirnov, Phys. Lett. B 598 (2004) 55, hep-ph/0406053.

[22] M. Czakon, J. Gluza, T. Riemann, Phys. Rev. D 71 (2005) 073009, hep-ph/0412164.

[23] M. Czakon, J. Gluza, K. Kajda, T. Riemann, Nucl. Phys. B (Proc. Suppl.) 157 (2006) 16-20, hep-ph/0602102.

[24] M. Czakon, J. Gluza, T. Riemann, Nucl. Phys. B 751 (2006) 1-17, hep-ph/0604101.

[25] R. Bonciani, A. Ferroglia, Two-loop QED Bhabha scattering, http://pheno.physik.uni-freiburg.de/ bhabha/.

[26] S. Actis, M. Czakon, J. Gluza, T. Riemann, Two-loop QED Bhabha scattering, http://www-zeuthen.desy.de/theory/ research/bhabha/bhabha.html/.

[27] S. Actis, A. Ferroglia, G. Passarino, M. Passera, C. Sturm, S. Uccirati, GraphShot, a FORM package for automatic generation and manipulation of one and two loop Feynman diagrams, unpublished.

[28] P. Nogueira, J. Comput. Phys. 105 (1993) 279.

[29] P. Nogueira, An introduction to QGRAF 2.0, ftp://gtae2.ist.utl.pt/pub/qgraf/.

[30] M. Tentyukov, J. Fleischer, Comput. Phys. Commun. 132 (2000) 124-141, hep-ph/9904258.

[31] J. Vermaseren, New features of FORM, math-ph/0010025.

[32] M. Czakon, DiaGen/IdSolver, unpublished. 
[33] S. Laporta, E. Remiddi, Phys. Lett. B 379 (1996) 283-291, hep-ph/9602417.

[34] S. Laporta, Int. J. Mod. Phys. A 15 (2000) 5087-5159, hep-ph/0102033.

[35] E. Remiddi, J. Vermaseren, Int. J. Mod. Phys. A 15 (2000) 725-754, hep-ph/9905237.

[36] T. Gehrmann, E. Remiddi, Nucl. Phys. B 580 (2000) 485-518, hep-ph/9912329.

[37] T. Gehrmann, E. Remiddi, Nucl. Phys. B 601 (2001) 248-286, hep-ph/0008287.

[38] S. Wolfram, The Mathematica Book, Wolfram Media/Cambridge Univ. Press, 2003.

[39] N. Usyukina, Teor. Mat. Fiz. 22 (1975) 300-306.

[40] E. Boos, A. Davydychev, Theor. Math. Phys. 89 (1991) 1052-1063.

[41] M. Czakon, Comput. Phys. Commun. 175 (2006) 559-571, hep-ph/0511200.

[42] M. Roth, A. Denner, Nucl. Phys. B 479 (1996) 495-514, hep-ph/9605420.

[43] S. Moch, P. Uwer, Comput. Phys. Commun. 174 (2006) 759-770, math-ph/0508008.

[44] S. Actis, M. Czakon, J. Gluza, T. Riemann, Nucl. Phys. B (Proc. Suppl.) 160 (2006) 91-100, hep-ph/0609051.

[45] G. 't Hooft, M. Veltman, Nucl. Phys. B 44 (1972) 189-213.

[46] C. Bollini, J. Giambiagi, Nuovo Cimento B 12 (1972) 20-25.

[47] R. Bonciani, P. Mastrolia, E. Remiddi, Nucl. Phys. B 661 (2003) 289-343, hep-ph/0301170.

[48] P. Mastrolia, E. Remiddi, Nucl. Phys. B 664 (2003) 341-356, hep-ph/0302162.

[49] R. Bonciani, P. Mastrolia, E. Remiddi, Nucl. Phys. B 676 (2004) 399-452, hep-ph/0307295.

[50] G. Burgers, Phys. Lett. B 164 (1985) 167.

[51] D. Bardin, P. Christova, M. Jack, L. Kalinovskaya, A. Olchevski, S. Riemann, T. Riemann, Comput. Phys. Commun. 133 (2001) 229-395, hep-ph/9908433.

[52] A. Arbuzov, M. Awramik, M. Czakon, A. Freitas, M. Grünewald, K. Mönig, S. Riemann, T. Riemann, Comput. Phys. Commun. 174 (2006) 728-758, hep-ph/0507146.

[53] B. Kniehl, M. Krawczyk, J. Kühn, R. Stuart, Phys. Lett. B 209 (1988) 337.

[54] J. Fleischer, J. Gluza, A. Lorca, T. Riemann, Eur. J. Phys. 48 (2006) 35-52, hep-ph/0606210.

[55] T. Becher, K. Melnikov, arXiv: 0704.3582 [hep-ph]. 